\title{
Structural Features of Human DJ-1 in Distinct Cys106 Oxidative States and their Relevance to its Loss of Function in Disease
}

Róbert Kiss ${ }^{1, *}$, Max Zhu ${ }^{2, *}$, Balázs Jójárt ${ }^{3}$, András Czajlik ${ }^{1}$, Katalin Solti ${ }^{1}$, Balázs Fórizs ${ }^{2}$, Éva Nagy ${ }^{1}$, Ferenc Zsila ${ }^{4}$, Tamás Beke-Somfai ${ }^{4}$, Gergely Tóth ${ }^{1,2}$

${ }^{1}$ MTA-TTK-NAP B - Drug Discovery Research Group - Neurodegenerative Diseases, Institute of Organic Chemistry, Research Center for Natural Sciences, Hungarian Academy of Sciences, Budapest, Hungary

${ }^{2}$ Cantabio Pharmaceuticals, Sunnyvale, California, USA

${ }^{3}$ Department of Chemical Informatics, Faculty of Education, University of Szeged, Boldogasszony sgt. 6 , Szeged 6725, Hungary

${ }^{4}$ Biomolecular Self-Assembly Group, Institute of Materials and Environmental Chemistry, Research Centre for Natural Sciences, Hungarian Academy of Sciences, Budapest, Hungary

* Both authors contributed equally to this manuscript.

Running Title: Structural Features of DJ-1 in Cys106 Oxidation-dependent States

To whom correspondence should be addressed: Dr. Gergely Tóth, MTA-TTK-NAP B - Drug Discovery Research Group - Neurodegenerative Diseases, Institute of Organic Chemistry, Research Center for Natural Sciences, Hungarian Academy of Sciences, Budapest, Hungary; phone: +36 1382 6618, email: toth.gergely@ttk.mta.hu

Keywords: DJ-1; Parkinson disease; oxidative stress; molecular dynamics; CSD; OCS

\section{ABSTRACT}

DJ-1 (PARK7) is a multifunctional protein linked to the onset and progression of a number of diseases, most of which are associated with high oxidative stress. The oxidation state of Cys106 of DJ-1 is believed to determine the specific functions of the protein in normal and disease conditions. Here we report molecular dynamics simulation and biophysical experimental studies on $\mathrm{DJ}-1$ in reduced (Cys106, $-\mathrm{S}^{-}$), oxidized (Cys106, $-\mathrm{SO}_{2}^{-}$), and over-oxidized (Cys106, $-\mathrm{SO}_{3}^{-}$) states. To simulate the different oxidation states of Cys106 in DJ-1, AMBER related force field parameters were developed and reported for 3-sulfinoalanine and cysteine sulfonic acid. Our studies found that the overall structure of DJ-1 in different oxidation states was similar globally, while it differed locally significantly, which have implications on its stability, function and its link to disease on-set. Importantly, the results suggest that over-oxidation may trigger loss of functions due to local structural modification in the Cys106 containing pocket of
DJ-1 and structurally destabilize the dimeric state of DJ-1, which is believed to be its bioactive conformation. Such loss of functions would result in reduced ability of DJ-1 to protect from oxidative stress insults and may lead to increased progression of disease.

\section{INTRODUCTION}

DJ-1 is a multifunctional protein directly linked to the onset and progression of a number of diseases [1], such as neurodegenerative diseases [2,3], stroke [4], type II diabetes [5] and cancer $[6,7]$. Mutations in the DJ-1 encoding gene (PARK7) can cause familial autosomal recessive early-onset Parkinson's disease (PD) [8]. DJ-1 is ubiquitously expressed including neurons and glial cells [9]. DJ-1 has been shown to have a wide range of physiological functions [1] including: redox sensor in oxidative stress, reactive oxygen species (ROS) quencher [10], molecular chaperone 
[11,12], protease [13], glyoxalase [14] and transcriptional regulator [1].

The DJ-1 monomer consists of 189 residues, which structurally fold into seven beta-strands and eight helices homologous to members of the ThiJ/PfpI family. Under physiological conditions it has been shown to form homodimers (Fig. 1A) but higher-order assemblies have been also identified in vitro [15] and in vivo [11,16]. DJ-1 contains a crucial cysteine residue (Cys106) that is buried deep in the putative binding site with a strained dihedral conformation. It has a depressed $\mathrm{p} K_{\mathrm{a}}$ of 5.4 [17] that makes this residue unusually reactive and thus sensitive to oxidation due to oxidative stress [18]. The side chain of Cys106 can be oxidized from thiolate (-S), to sulfinate ($\left.\mathrm{SO}_{2}{ }^{-}\right)$, or sulfonate $\left(-\mathrm{SO}_{3}{ }^{-}\right)[19,20]$, referred to as the reduced, oxidized and over-oxidized forms of DJ-1, respectively in this article (Fig. 1B). The mono-oxidized, sulfenate ( $\left.\mathrm{SO}^{-}\right)$state of $\mathrm{DJ}-1$ is assumed to be transient, while the reduced and oxidized states are stable and can have a variety of distinct physiological functions [1].

The oxidation state of Cys106 determines the specific functions of DJ-1 [1]. Particularly, the oxidation of Cys106 to the sulfinate state has been shown to be required for its neuroprotective function [21], while the sulfonate state of Cys 106 has been linked to loss of function $[1,2,22,23]$. The oxidative damage of DJ-1 due to over-oxidation was linked to PD [24] and observed in postmortem brain samples in PD and Alzheimer's disease (AD) patients [25] suggesting that the impaired functions of the over-oxidized DJ-1 may play an important role in the onset and progression of PD [26].

Several structural studies, primarily experimental, have been published on DJ-1. At the time of writing this manuscript, a query for "DJ-1" in PDB (Protein Data Bank, http://rcsb.org) retrieves 41 records. Due to the extreme sensitivity of Cys106 to oxidation, most available X-ray structures capture DJ-1 in its oxidized state. There are difficulties in keeping recombinant DJ-1 in the reduced form due to increased oxidative risks, as a result of alkaline $\mathrm{pH}$ and heavy metal ions presence during purification stages [17]. In addition, the crystallographic procedure further promotes oxidation of Cys106 [21]. Consequently, some reported reduced DJ-1 structures may actually be in an oxidized state [27]. In summary, experimental structural information on reduced apo DJ-1 is limited whereas there is no available crystal structure of over-oxidized DJ-1.

To improve our understanding of structural and biophysical characteristics of the different oxidation states of DJ-1 molecular dynamics (MD) simulation can be applied, especially as experimental information is limited on the reduced and over-oxidized states. Although short MD simulations were reported on DJ-1, these mainly focused on mutations destabilizing the dimer interface [28,29].

The main aims of this study are to analyze and identify structural features of the reduced, oxidized and over-oxidized forms of DJ-1 and elucidate their role in its physiological and protective functions in disease. Moreover, our results may aid the identification and development of state-specific small-molecule ligands to DJ-1, which could have different therapeutic and diagnostic potential. Here we report both MD and experimental investigation on the different Cys106 oxidation states of DJ-1, which can provide insights into the function and loss-of-function of DJ-1 and their implications to the onset and progression of PD and other diseases.

\section{MATERIALS AND METHODS}

DJ-1 X-ray Structure Analysis. DJ-1 X-ray structures were prepared by first separating individual DJ-1 monomers from dimers and oligomers. From the resulting 61 monomers, residues before Ser3 and after Leu187 were eliminated and the residues were renumbered to obtain a consistent set of structures. Alignment was performed using the McLachlan algorithm [30] as implemented in the program ProFit 
[Martin, A.C.R. and Porter, C.T., (http://www.bioinf.org.uk/software/profit/) ProFit version 3.1] $\mathrm{C} \alpha$ atoms of all 61 DJ-1 monomers were aligned and an average alignment structure was generated. The RMSD was calculated by Profit for each DJ-1 monomer as the distance of the $\mathrm{C} \alpha$ atoms from the average DJ-1 structure.

Parametrization of different oxidation states of cysteine. We followed the protocol used for phospho-amino acid parameter development [31]. Our model systems were the ACE and NME capped dipeptides. All derivatives were considered in their negatively charged form. Two backbone conformers were built: one in $\alpha$-helix $\left(\varphi=-60.0^{\circ}\right.$ and $\psi=-45.0^{\circ}$ ) and the other one in extended conformation $\left(\varphi=-135.0^{\circ}\right.$ and $\left.\psi=135.0^{\circ}\right)$. For the obtained structures geometry optimization at the $\mathrm{HF} / 6-31 \mathrm{G}^{*}$ level of theory was performed with Gaussian09 program package [32]. After optimization the structures were subjected to molecular electrostatic potential (MEP) calculations at the same level of theory using Gaussian09. Point charge fitting was performed with resp module of AMBER14 [33]. During MEP calculations two orientations were considered according to the standard protocol [34]. For amide $\mathrm{N}$ and $\mathrm{H}$ atoms and for carbonyl $\mathrm{C}$ and $\mathrm{O}$ atoms the following charges were constrained: -0.4157 , $0.2719,0.5973$ and -0.5679 , respectively. In case of CSD and OCS residues further dihedral restraints were applied to avoid $\mathrm{H}$-bond formation between SOx groups and backbone atoms.

For general amber force field (GAFF) [35] parameter assignment the antechamber [36] module from AMBER14 [33] distribution was used and the following atom types were assigned automatically for sulfur: $s 4$ (S with three connected atoms) and $s 6(\mathrm{~S}$ with four connected atoms) for CSD and OCS, respectively. For side chain oxygen(s) $o$ atom type (oxygen with one connected atom) was assigned automatically. For backbone and for $\beta$ carbon atoms parameters from AMBER99SB [37] force field were assigned.
Protein structures with $<1.5 \AA$ resolution containing CSD and OCS residues were collected from the Protein Data Bank (www.rscb.org). PDB codes of the investigated protein structures are available in SI (Table S2). Bond distances (CB$\mathrm{SG}, \mathrm{SG}-\mathrm{ODx}$, where $\mathrm{x}=1,2,3$ ) and angles (CACB-SG, CB-SG-ODx, ODx-SG-ODx) were calculated and compared to the equilibrium values in the GAFF force field.

In order to verify the quality of the calculated charges and the parameters obtained from GAFF, 2D rotational profiles of the side chain of CSD and OCS were calculated at the HF/6-31G* and AMBER99SB/GAFF level of theory. Structures for the two backbone conformers were generated by the tleap module of AMBER 14. Angles $\chi_{1}$ and $\chi_{2}$ were varied between -180 and 180 by $20^{\circ}$ increments. Thereafter all structures were optimized at the AMBER99SB/GAFF level of theory (gas phase). The optimized structures were subjected to geometry optimization at the $\mathrm{HF} / 6-31 \mathrm{G}^{*}$ level of theory. During geometry optimization $\varphi, \psi, \chi_{1}$ and $\chi_{2}$ were restrained (AMBER99SB/GAFF) or fixed (HF/6-31G*). As a further comparison and validation the same side chain dihedral scan was performed for aspartate (ASP), protonated histidine (HIP) and leucine (LEU) residues.

DJ-1 structure preparation for MD simulation. The structure 3SF8 PDB structure [27] was pre-processed for the simulations by the MOE (ver. 2013.0802) Structure preparation module. After removing waters and counterions, we eliminated the artificial 'Leu-Glu' motif at the Cterm and the first and the last residues of DJ-1 as they were not well resolved by X-ray crystallography leaving a 187-residue DJ-1 homodimer as our base system. We assumed that in a solvent environment, the oxidation state of the Cys106 in both subunits is the same and thus built our system accordingly: we generated structures where both Cys106 residues were in thiolate $\left(-\mathrm{S}^{-}\right)$, sulfinate $\left(-\mathrm{SO}_{2}^{-}\right)$and sulfonate $\left(-\mathrm{SO}_{3}{ }^{-}\right)$forms. Starting from the thiolate system, we built up two 
extra oxygens using available information in X-ray structures capturing the $\mathrm{SO}_{2}{ }^{-}$form of Cys106. The geometry of the $\mathrm{SO}_{2}{ }^{-}$form defined the position of the third oxygen yielding the $\mathrm{SO}_{3}{ }^{-}$form. A short local minimization was carried out on side chain and $\mathrm{Ca}$ atoms of Cys106 and Glu18 using MMFF94 force field by default settings for each system in MOE. Protonate $3 D$ module was applied to generate the most probable protonation and tautomer states of all residues. Default settings were applied except for residues Glu18 and Cys106 where we relied on the available experimental data [18] and assumed Glu18 to be protonated and Cys106 to be deprotonated.

Explicit-solvent molecular dynamics simulations of DJ-1. We performed molecular dynamics (MD) simulations of the prepared DJ-1 system using GROMACS [38]. The production run of the simulation was $200 \mathrm{~ns}$, using an integration step of $2 \mathrm{fs}$, at $298 \mathrm{~K}$ with the AMBER99SB-ILDN force field and the TIP3P water model [37], which has been shown to reproduce NMR parameters in MD simulations of other peptides and proteins with good accuracy [39]. The prepared DJ-1 homodimer with charged termini was first energy-minimized by steepest descent for 10,000 steps in vacuo, which was then placed in a cubic box of $9.3 \times 9.3 \times 9.3 \mathrm{~nm}^{3}$ in periodic boundary conditions, with about 7,000 water molecules and $\mathrm{Na}^{+} / \mathrm{Cl}^{-}$counterions for neutralizing the net charge of the system at physiological salt concentration. This system was then further energy-minimized by steepest descent for 50,000 steps. It was followed by equilibration in the NVT ensemble from $0-100 \mathrm{~K}$ for $500 \mathrm{ps}$ and in the NPT ensemble from $100-298 \mathrm{~K}$ for $1 \mathrm{~ns}$, before the production run.

We verified convergence of simulations from different measures including the evolutions of Ca RMSD, radius of gyration, SASA, and energy profiles. By these measures, we judged that from $100 \mathrm{~ns}$ the simulations appear to have converged relatively well. The main analysis performed in this report was taken from the 100-
$200 \mathrm{~ns}$ trajectories of the three respective simulations. Convergence data can be found in SI.

MM/GBSA calculation. The binding free energy calculations between the two monomer subunits were performed with the MMPBSA.py [40] module implemented in AMBER14 software package [33]. Implicit solvent model $5(\mathrm{igb}=5)$ was used with mbondi2 radii sets [41] and the non-polar term was calculated using the LCPO method [42] with a surface tension value of 0.005 $\mathrm{kcal} / \mathrm{mol} / \AA^{2}$ and salt concentration set to 0.15 $\mathrm{mol} / \mathrm{dm}^{3}$. In order to eliminate too many similar structures only 20,000 structures from 100-200 ns, with an interval of $5 \mathrm{ps}$, were considered. Residues forming intermonomer interactions were identified as follows: in each frame of the simulations we selected residues from chain $\mathrm{A}$ that were closer than $4.5 \AA$ to any residue in chain $\mathrm{B}$ and vice versa. Residues with occurrence of at least $10 \%$ in either of the simulations were considered to be part of the monomer-monomer interface.

DJ-1 expression and purification. DJ-1 was overexpressed in E. coli Rosetta (DE3) strain as glutathione S-transferase (GST) fusion protein. At optical density $\left(\mathrm{A}_{600}\right)$ of 0.6 cells was induced by $0.1 \mathrm{mM}$ isopropyl- $\beta$-D-thiogalactopyranoside (IPTG) for overnight at $18{ }^{\circ} \mathrm{C}$ and harvested by centrifugation and frozen at $-80{ }^{\circ} \mathrm{C}$. Pellets were thawed and resuspended in lysis buffer $(50 \mathrm{mM}$ Tris- $\mathrm{HCl}, 100 \mathrm{mM} \mathrm{NaCl}, 0,1 \%$ Igepal, $0.5 \mathrm{mM}$ DTT, $\mathrm{pH}=8$ ) and broken by sonication. After a further centrifugation the lysate was purified with a GSTrap ${ }^{\mathrm{TM}} 4 \mathrm{~B}$ column (GE Healthcare) using an elution buffer containing $30 \mathrm{mM}$ GSH. The GSTtag was then cleaved by PreScission protease (GE Healthcare). In the final step the protein was eluted in a GSTrap ${ }^{\mathrm{TM}} 4 \mathrm{~B}$ column to get rid of the GST-tag and protease, flow-through was collected and dialysed into a buffer containing $50 \mathrm{mM}$ Tris$\mathrm{HCl}, 100 \mathrm{mM} \mathrm{NaCl}, 1 \mathrm{mM}$ DTT (pH=7.4).

\section{Liquid chromatography - mass} spectrometry. DJ-1 samples were characterized by LCMS using a Shimadzu LCMS-2020 equipped by a Phenomenex Aeris Widepore XB-C8 column. 
$5 \mu \mathrm{L}$ of DJ-1 samples $(30 \mu \mathrm{M})$ were injected into eluents $\mathrm{A}(10 \mathrm{mM}$ ammonium-formiate $(\mathrm{pH}=3)$ in distilled water) and $\mathrm{B}(10 \mathrm{mM}$ ammoniumformiate $(\mathrm{pH}=3)$ in $90 \%$ acetonitrile and $10 \%$ distilled water) with a flow rate of $0.3 \mathrm{~mL} / \mathrm{min}$ and the following gradient: $25-50 \% \mathrm{~B}$ during $10 \mathrm{~min}$, then $25 \% \mathrm{~B}$ for $3 \mathrm{~min}$. Temperature of column was set to $50^{\circ} \mathrm{C}$. Spectra were acquired in the positive mode in the $50-2000 \mathrm{~m} / \mathrm{z}$ range using DUIS ionization and Profile MS method. Positive spectra were analyzed. Mass spectra were processed using our in-house developed program: an implementation of the ZSCORE algorithm [43]. Briefly, mass spectra are converted to zero-charge mass spectra by ranking possible charges for each individual $\mathrm{m} / \mathrm{z}$ peaks. Charges with multiple identified isotopic peaks in the mass spectra get higher ranks. After the most probable charge states have been identified the $\mathrm{m} / \mathrm{z}$ values were converted to zero-charge mass values by deducting mass of protons and multiplied by the charge. In the resulting zero-charge mass spectra, the ratio of the most dominant molecular weight samples is determined. The most dominant molecular weights of the reduced, oxidized and over-oxidized DJ-1 samples used in the experiments are summarized below:

Reduced sample: 20303.3 (30.7\%), 20332.8 (14.2\%), 20352.8 (6.1\%), $20371.4 \quad$ (3.6\%), $20391.1 \quad(2.9 \%) . \quad$ Oxidized sample: $20336.3(28.4 \%), \quad 20359.2(7.2 \%), \quad 20381.5$ (6.7\%), 20399.9 (4.3\%), 20420.3 (2.8\%). Overoxidized sample: 20346.7 (17.2\%), 20378.7 (7.8\%), 20398.8 (6.0\%), 20329.3 (5.2\%), 20360.9 (5.0\%). The reduced sample contained a reduced/oxidized DJ-1 ratio of $68: 32$. In the oxidized and over-oxidized samples virtually no reduced form could be detected. The oxidized sample contained predominantly the sulfinate form (2 additional oxygens), while in the over-oxidized DJ-1 sample the sulfonate form (3 additional oxygens) dominated and some minor components with 2, 5, 6 oxygens.
Dynamic Light Scattering. Measurements were taken at $25{ }^{\circ} \mathrm{C}$ using a Malvern Zetasizer Nano ZS instrument equipped with a thermostatted cell. DJ-1 in $20 \mathrm{mM}$ Tris buffer $\mathrm{pH} 7.5$, at concentrations of $1 \mathrm{mg} / \mathrm{mL} \quad(50 \mu \mathrm{M})$ was centrifuged at $10,000 \mathrm{rpm}$ for $10 \mathrm{~min}$ at $4{ }^{\circ} \mathrm{C}$. The samples were filtered through $0.2 \mu \mathrm{m}$ filter before the measurements. $100 \mu \mathrm{L}$ of the supernatants were added to the cuvette, and the light scattering intensity was collected 30 times at an angle of $90^{\circ}$ using a $10 \mathrm{sec}$ acquisition time. The correlation data were exported and analyzed using the nano DTS software (Malvern Instruments).

Different Scanning Calorimetry. Experiments were carried out using reduced, oxidized and over-oxidized DJ-1 at $10 \mu \mathrm{M}$ concentration. DJ-1 samples were dialyzed into HEPES buffer (10 mM HEPES, $5 \mathrm{mM} \mathrm{NaCl}, \mathrm{pH}$ $=7.4)$ as well as phosphate $(20 \mathrm{mM}$ potassiumphosphate, $\mathrm{pH}=7.4$ ) at $4{ }^{\circ} \mathrm{C}$. VP-DSC microcalorimeter (Microcal) was used for all experiments over a temperature range of 25-100 ${ }^{\circ} \mathrm{C}$ with a scan rate applied was $60^{\circ} \mathrm{C} / \mathrm{h}$. Prior to the DSC experiments the samples and the reference solution were degassed for at least 15 min at room temperature. For all measurements three reference buffer and duplicate DJ-1 sample scans were collected. Data were analyzed subtracting the thermogram of the reference buffer as well as baseline by Microcal Origin 5.0 software package supplied with the instrument. Reversibility of the thermal transition of DJ-1 was also examined by doing a second scan after cooling down from the first one. The observed $\mathrm{T}_{\mathrm{m}}$ values differed by less than $0.2{ }^{\circ} \mathrm{C}$ in parallel DSC experiments for all type of DJ-1 samples.

\section{Circular dichroism and $U V$ absorption} spectroscopic measurements. DJ-1 solutions were dialized into $10 \mathrm{mM}$ pH 7.2 HEPES buffer $(5 \mathrm{mM}$ $\mathrm{NaCl}, 1 \mathrm{mM}$ dithiothreitol). $\mathrm{CD}$ and UV absorption data were acquired at $25 \pm 0.2{ }^{\circ} \mathrm{C}$ on a JASCO J-715 spectropolarimeter equipped with a Peltier thermostat. UV spectra were obtained by conversion of the high tension (HT) voltage 
applied to the photomultiplier tube into absorbance units. Far-UV CD spectra were monitored in continuous scanning mode between 205 and 260 $\mathrm{nm}$ at a rate of $50 \mathrm{~nm} / \mathrm{min}$, with a step size of 0.2 $\mathrm{nm}$, response time of $2 \mathrm{sec}$, five accumulations, 2 $\mathrm{nm}$ bandwidth, using a $0.1 \mathrm{~cm}$ path-length quartz cuvette (Hellma, USA). Near-UV CD curves were recorded from 250 to $350 \mathrm{~nm}$ in a $1 \mathrm{~cm}$ pathlength quartz cell at a rate of $50 \mathrm{~nm} / \mathrm{min}$, with a step size of $0.2 \mathrm{~nm}$, response time of $2 \mathrm{sec}$, five accumulations, $1 \mathrm{~nm}$ bandwidth. CD curves of protein samples were corrected by spectral contribution of blank buffer solution. CD spectra were plotted in mean residue molar ellipticity units (deg $\mathrm{cm}^{2} \mathrm{dmol}^{-1}$ residue ${ }^{-1}$ ) calculated by the following equation: $[\Theta]=\Theta_{\text {obs }} /(10 \mathrm{ncl})$ where $[\Theta]$ is the mean residue molar ellipticity, $\Theta_{\mathrm{obs}}$ is the measured ellipticity (mdeg) as a function of wavelength, $n$ is the number of the protein residues, $c$ is the molar concentration of the protein, and $l$ is the optical path length $(\mathrm{cm})$. Secondary structure analysis of DJ-1 using CD spectroscopic data was carried out by using the K2D software [44].

\section{RESULTS}

DJ-1 X-ray Structure Analysis. As a reference for the interpretation of the simulation results to be described, the available experimental structural data on DJ-1 were analyzed. DJ-1 X-ray structures available at the time of the study were collected and analyzed by root-mean-squareddeviation (RMSD), secondary structure content, hydrodynamic diameter and backbone dihedrals of the critical Cys106 residue. Structures holding any mutations or containing ligands/metal ions were excluded from the analysis to rule out their effects on the structural properties under study.

Average RMSD of backbone atoms from the average alignment structure ranged between 0 $0.3 \AA$ for $>95 \%$ of the residues (Fig. 1C). Larger fluctuations were observed in the $\mathrm{N}$-terminal residue Ser3, a short water-exposed sequence at the end of the second helix (Lys62 - Pro66) and
Asn76 at the entrance of the Cys106 binding site. $\alpha$-helical and $\beta$-sheet contents of the X-ray structure set were also analyzed and only minor variations in the helical content predominantly at the beginning and at the end of helices were found (Fig. 1D).

The hydrodynamic diameter $\left(\mathrm{D}_{\mathrm{h}}\right)$ of the analyzed X-ray structures ranged between 5.46 $5.65 \mathrm{~nm}$ with an average value of $5.54 \mathrm{~nm} \pm 0.056$ (S.D.) as calculated by HYDROPRO [45] for Ser3-Leu187 of DJ-1. Full list of $D_{h}$ values are given in Table $\mathrm{S} 3$.

The backbone dihedrals of the critical Cys106 residue were analyzed, which ranged between $55^{\circ}-80^{\circ}$ and $-90^{\circ}-\left(-115^{\circ}\right)$, respectively for $\Phi$ and $\Psi$ angles (Fig. 2). These ranges have been shown to be rarely populated in cysteine residues [46]. Moreover, $\Psi$ angles in cysteines between $-90^{\circ}$ and $-150^{\circ}$ were suggested to result in an energetically strained backbone conformation required for enzymatic reactions [46].

On the basis of the available DJ-1 X-ray structures, the oxidation of DJ-1 happens in a specific structural configuration, because the oxygen positions are occupied in a well-defined order as suggested by Wilson et al. [47]. Some DJ1 X-ray structures capture the transient monooxidized form of Cys106, where the extra oxygen forms hydrogen bonds with the backbone $\mathrm{NH}$ groups of Ala107 and Gly75 suggesting that this position is energetically most favored and is occupied first during Cys106 oxidation. X-ray structures containing the $-\mathrm{SO}_{2}^{-}$form of Cys106 show that the second oxygen interacts with Glu18, another important residue for DJ-1 function, which is expected to be protonated at physiological $\mathrm{pH}$. There is currently no X-ray structure available for over-oxidized DJ-1 containing Cys106 in $-\mathrm{SO}_{3}{ }^{-}$ form. Nevertheless, the geometric restraints of the oxygen positions in the $-\mathrm{SO}_{2}^{-}$form determine the initial position of the third oxygen pointing toward His126.

Novel Parameters for Oxidized Forms of Cysteine. No AMBER related molecular 
mechanics force field parameters are available for oxidized Cys residues. In order to simulate the different oxidation states of Cys106 in DJ-1, AMBER related force field parameters were developed for the following modified cysteine residues: 3-sulfinoalanine (CSD) and cysteine sulfonic acid (OCS) (Fig. 1B). Geometric data and calculated charges obtained from the parametrization are summarized in Table 1 and 2, while validation results of the parametrization are provided in Table $\mathrm{S} 1$.

The equilibrium values of the analyzed distances and angles from the GAFF parameter set were found to be close to the values obtained from high resolution crystal structures (see Table S2). Calculated values for CSD and OCS in the dihedral scan were similar to those found for standard amino acid residues (Table S1). Therefore these parameters were used in our simulation studies without further modifications. These force field parameters for CSD and OCS can be applied in MD of other polypeptide and protein systems containing oxidized cysteine residues, such as phosphatases and peroxiredoxins [48,49].

DJ-1 MD simulations. Three DJ-1 homodimer systems, with Cys106 in reduced, oxidized and over-oxidized states in both monomers, were prepared and simulated by MD solvated in explicit water for $200 \mathrm{~ns}$. Time series of root-mean-squared-deviation (RMSD) to reference structures, radius of gyration ( $R g$ ), solvent accessible surface area (SASA) and MM/GBSA monomer-monomer interaction energy profiles indicated high degree of convergence after $100 \mathrm{~ns}$ (see Fig. S1-4). Note that we also refer the first and second monomer in the homodimer system as chain A and chain B respectively in the remainder of this article.

First, the interactions formed by the oxygens of $-\mathrm{SO}_{2}{ }^{-}$and $-\mathrm{SO}_{3}{ }^{-}$forms of Cys106 were analyzed. As described in the previous section, the first oxygen occupied by Cys106 during oxidation forms interactions with the backbone $\mathrm{NH}$ groups of Ala107 and Gly75. In the simulations, Cys106 in reduced DJ-1 rarely formed interactions with these residues. On the other hand, these interactions existed in most of the simulation time in oxidized DJ-1 as well as in over-oxidized DJ-1 (the corresponding heavy atom distances are plotted in Fig. 3A-D). Interactions formed by the second oxygen were also analyzed. In the simulations, Glu18 is mainly coordinated by Cys106, however certain oxidation-state dependent differences were observed (Fig. 3E-H). The distance between the sulfur atom of Cys106 and the protonated side chain oxygen of Glu18 in reduced DJ-1 was longer $(\sim 3-4 \AA)$ than the distance between the side chain oxygen atoms of $\mathrm{SO}_{2}{ }^{-}$and $-\mathrm{SO}_{3}{ }^{-}$of Cys106 in oxidized and overoxidized DJ-1 (2.7 $\AA$ ). In oxidized DJ-1, the interaction with Glu18 was particularly stable. In contrast, in chain A of reduced DJ-1 this interaction was lost and it did not reform later on. Additionally, the over-oxidized form showed some minor fluctuations in chain $\mathrm{A}$, but the corresponding heavy atom distance remained below $3.5 \AA$. Interestingly, in the over-oxidized system the distance between Glu18 and Cys106 correlated with the distance between the protonated side chain oxygen of Glu18 and side chain oxygen of Glu15. In fact, Glu18 and Glu15 were getting closer as the Glu18-Cys106 distance was increasing suggesting that Glu15 and Cys106 were competing for Glu18.

The third oxygen of over-oxidized Cys106 of chain B formed an interaction with the backbone $\mathrm{NH}$ amide group of Gly157 of the same chain, while no such interaction was present in chain A (Fig. 3I-J). In addition, during MD of over-oxidized DJ-1 the third oxygen pointed toward His126 and the imidazole $\mathrm{NH}$ group of His126 was in close proximity to the side chain oxygens of Cys106 and for a short period of time, in chain B, an H-bond was formed (Fig. 3K-L). Interactions with backbone $\mathrm{NH}$ group of Gly157 or imidazole NH group of His126 could not be detected in the reduced or oxidized states of DJ-1. 
Besides the direct interactions formed by the extra oxygen atoms in oxidized and overoxidized DJ-1, a number of indirect effects of oxidation were also observed in the simulations. First, the interaction profile of the backbone heteroatoms of Cys106 was analyzed. The backbone amide $\mathrm{NH}$ group of Cys106 formed a stable interaction with backbone amide oxygen of Ser155 in all oxidation states (see Fig. S5 A and B in SI). In contrast, the other Cys106 backbone stabilizing interaction between the backbone oxygen of Cys106 and backbone nitrogen of His126 remained intact in the reduced and oxidized states only (see Fig. S5 C and D).

The backbone dihedrals of Cys106 in the simulations ranged between $40^{\circ}-80^{\circ}$ and $-60^{\circ}$ - ($140^{\circ}$ ), respectively for $\Phi$ and $\Psi$ angles (Fig. 2). The $\Psi$ angle decreased with the oxidation state of Cys106 in both chains, and was more pronounced in chain B. The $\Phi$ angles showed less oxidationstate dependent differences. The $\chi 1$ angle of the side chain of Cys106 remained stable for the majority of the simulations in chain A, being slightly lower in average value for the overoxidized compared to reduced and oxidized forms (see Fig. S6), However, in chain B of the reduced and over-oxidized DJ-1 there were significant differences in the $\chi 1$ angle compared to chain A.

At the intermonomer level, Cys53 of chain $\mathrm{A}$ and $\mathrm{B}$ can be found in close proximity in most DJ-1 X-ray structures and has been shown to be able to form a disulfide bridge under oxidative conditions in vitro [26]. Although this form of DJ1 was not investigated in this study, the distances between the two sulfur atoms (as thiols) were monitored (see Fig. S7). The distributions showed that there were two major populations at 3.5 and $5.5 \AA$ distances indicating that under oxidative stress the sulfur atoms can get sufficiently close to form a disulfide bond.

The effect of oxidation on the quaternary structure of DJ-1 was also analyzed. The calculated hydrodynamic diameter $\left(\mathrm{D}_{\mathrm{h}}\right)$ of the DJ1 dimer was slightly larger in the over-oxidized form $\left(D_{h}=5.614 \mathrm{~nm} \pm 0.022\right.$ (S.D.)), while the reduced $\left(\mathrm{D}_{\mathrm{h}}=5.604 \mathrm{~nm} \pm 0.022\right.$ (S.D.)) and oxidized $\left(D_{h}=5.604 \mathrm{~nm} \pm 0.022\right.$ (S.D.) forms were indistinguishable (see Fig. S8). Subsequently, the binding free energy between the two monomers was calculated using MM/GBSA calculations. The total intermonomer energies were significantly higher in the over-oxidized system $(-105.6 \mathrm{kcal} / \mathrm{mol})$ compared to the reduced $(-111.8 \mathrm{kcal} / \mathrm{mol})$ and oxidized $(-113.3 \mathrm{kcal} / \mathrm{mol})$ systems (see Fig. S9), suggesting that overoxidation of DJ-1 weakens the strength of the interaction between the two monomers and thus destabilizes the dimer.

The involvement of residues at the dimer interface (within $4.5 \AA$ of the other chain) was also evaluated. 80 residues were found with $10 \%$ or higher occurrence at the dimer interface in any one of the simulated systems. Significant differences were observed in the occurrences for some residue. In particular, residues at the $\mathrm{C}$-terminal of chain A and in the proximity of His126 in chain B were less frequently involved in monomermonomer interactions in the over-oxidized form compared to the reduced and oxidized forms (see Table S4). The binding energies for every possible residue pair of the 80 interfacing residues were calculated. Residues with the highest contribution to the differences in the over-oxidized compared to the reduced and oxidized states are highlighted in Fig. 4 (full list of per residue contributions are provided in Table S5). In line with these data, residues at the $\mathrm{C}$-terminal of chain $\mathrm{A}$ and in the His 126 region of chain $\mathrm{B}$ were found to contribute significantly to the intermonomer energy differences between over-oxidized and reduced/oxidized states. In particular, an intermonomer interaction between the backbone amide oxygen atom of Pro184 in chain A and imidazole NH group of His126 in chain B seemed to play a critical role in maintaining the dimer structure of DJ-1. The interaction remained stable except for the over-oxidized system (see Fig. S10), where it was lost during the first 20-30 ns of the 
simulation. In parallel Pro184 of chain A formed a H-bond with the backbone NH group of Gly159 in chain B. Similar interaction could not be observed in either the reduced or oxidized DJ-1 systems.

The SASA of DJ-1 in the different oxidation states also differed: the over-oxidized state had a slightly larger solvent exposed area, while the reduced and oxidized systems had similar SASA (see Fig. S9B). Some of the most prominent changes could be observed for residues at the $\mathrm{C}$ terminal of chain A and the His126 region of chain B (Fig. 4) (see also Table S6).

The secondary structure content was also monitored during the simulations. A small overall decrease in the $\alpha$-helical content was observed in over-oxidized DJ-1, and in parallel the ratio of unstructured content increased (see Fig. S11 in $\mathrm{SI})$. At the residue level, the C-terminal of chain A (residues 179-182) and the helix following His126 in chain B (residues 127-137, excluding Lys130 where the helical propensity increased) showed the most prominent differences (Fig. 5). The decrease of the $\alpha$-helical content in these regions were -26.5 $\% \pm 11.5$ (S.D.) and $-21.9 \% \pm 12.3$ (S.D.) when compared to oxidized and reduced DJ-1, respectively (see Fig. S12).

Comparison of the Cys106 binding sites. The SASA of the pocket in which Cys106 is located was compared in all simulated DJ-1 systems. The pocket in chain B of the overoxidized DJ-1 was extended (Fig. 6) compared to that of the reduced and oxidized DJ-1. The average SASA for residues within $6 \AA$ of Cys 106 of chain B was $99.07 \AA^{2}, 96.77 \AA^{2}$ and $110.39 \AA^{2}$ for reduced, oxidized and over-oxidized DJ-1, respectively. This extension in the over-oxidized form involved residues Ala183, Pro184 and Val186 in chain A and His126, Arg145, Arg156, Gly157, Pro158 in chain B. The SASA contributions of these residues were $30.28 \AA^{2}$, $30.00 \AA^{2}, 39.37 \AA^{2}$ for reduced, oxidized and overoxidized DJ-1, respectively. These results suggest that a significant difference may be promoted in the Cys106 containing pocket upon over-oxidation of DJ-1.

Hydrodynamic diameter of DJ-1. The hydrodynamic diameter of DJ-1 was determined to be $5.36 \pm 0.18,5.40 \pm 0.14,5.61 \pm 0.13 \mathrm{~nm}$, respectively for reduced, oxidized and overoxidized DJ-1 by dynamic light scattering (DLS). These values agree well with the calculated ones from the available X-ray structures as well as from our MD simulations (Table 3 ).

Thermal stability of DJ-1 measured by DSC. The thermal stability of the reduced, oxidized, and over-oxidized states of DJ-1 were determined by measuring the melting temperature $\left(\mathrm{T}_{\mathrm{m}}\right)$ of the proteins using differential scanning calorimetry (DSC). As reported in previous publications [50] the denaturation of all forms of DJ-1 was irreversible in both buffer systems tested. The $\mathrm{T}_{\mathrm{m}}$ value of oxidized DJ-1, $75.0{ }^{\circ} \mathrm{C}$, was significantly higher compared to that of reduced DJ-1, $62.2{ }^{\circ} \mathrm{C}$, in our HEPES buffer system similarly as reported by Lin et. al. [51]. Furthermore, for the first time, we observed that over-oxidized DJ-1 had similar thermal stability based on the measured $\mathrm{T}_{\mathrm{m}}, 75.1{ }^{\circ} \mathrm{C}$, as the oxidized form of DJ-1 (Fig. 7A).

Evaluation of DJ-1 secondary structure by CD spectroscopy. Far-UV CD spectra of the reduced and oxidized forms of DJ-1 exhibit two extrema that correspond to the $n-\pi^{*}(219 \mathrm{~nm})$ and $\pi-\pi^{*} \quad(211 \mathrm{~nm})$ transitions of the amide chromophores (Fig. 7B). The high qualitative and quantitative similarities between the $\mathrm{CD}$ curves of the reduced and oxidized samples refer to that chemical modification of the Cys106 residue does not affect significantly the overall secondary structure of DJ-1. This conclusion is in a full concordance with the percentages of secondary structure elements calculated from the CD data, which was found to be identical for each sample (Fig 7B). Deconvolution of the spectra employing the K2D algorithm predicted $37 \%$-helix content that agrees well with previous $\mathrm{CD}$ spectroscopic 
results [52]. A fuller description of CD spectra can be found in SI.

\section{DISCUSSION}

Novel simulation parameters for CSD and OCS. Cys106 in DJ-1 is sensitive to oxidation and thus can adopt different oxidation states, such as reduced $\left(-\mathrm{S}^{-}\right)$, oxidized $\left(-\mathrm{SO}_{2}{ }^{-}\right)$and over-oxidized $\left(-\mathrm{SO}_{3}{ }^{-}\right)$forms. Since no suitable force field parameters for oxidized cysteine were available in the literature, we developed them for the purpose of this study. These can hopefully facilitate, for the wider research community, the studies of other protein systems such as caspases, phosphatases and peroxiredoxins that frequently contain active site cysteines in different oxidation states [48,49,53].

Higher thermal stability of oxidized and over-oxidized DJ-1 compared to reduced DJ-1. Similarly to other studies reported in the literature [50,51], our results showed that the oxidized form of DJ-1 had substantially higher thermal stability compared to the reduced form. We show however for the first time that the $T_{m}$ of the over-oxidized form of DJ-1 is similar to that of the oxidized form. The increased thermal stability of oxidized and over-oxidized DJ-1 is suggested to be a consequence of the additional and stronger $\mathrm{H}$ bonds formed by the additional oxygen atoms of the oxidized forms vs. the sulfur atom of reduced Cys106. In particular, the $\mathrm{SO}_{2}^{-}$oxygens formed interactions with Ala107 and Gly75 in both oxidized and over-oxidized DJ-1, which were otherwise missing in reduced DJ-1 (Fig. 3A-D). The strength of these interactions is reflected by the fact that in the X-ray structures that contain mono-oxidized DJ-1, the oxygen occupies the position where it forms interactions with Ala107 and Gly75 instead of the other position where it could interact with Glu18. In chain B of overoxidized DJ-1 the interactions with Ala107 and Gly75 showed fluctuations and in their absence other stabilizing H-bonds were observed with Gly157 and His126 (Fig. 3I-L). In addition, we showed in the simulations that in oxidized and over-oxidized DJ-1 the interaction between Cys106 and Glu18 was stronger, reflected by the shorter interatomic distances (Fig. 3E-F). Similar stabilizing effect of the Cys106 side chain oxygens has been suggested for Drosophila DJ-1 $\beta$ [51].

General differences between oxidized and over-oxidized DJ-1. Our experimental results indicate an overall similar structure and stability of the oxidized and over-oxidized states of DJ-1. In particular, the thermal stability measured by DSC, the secondary structure profile assessed by $C D$ spectroscopy and the hydrodynamic diameter obtained by DLS yielded similar results. In line with these experimental results, the simulations showed only smaller differences at the full protein level. The calculated hydrodynamic diameter was slightly higher and the $\alpha$-helical content was slightly lower for the over-oxidized form, however, these differences were smaller than the detection limits in the experiments. On the other hand, several notable local differences were identified by the simulations.

Local differences between oxidized and over-oxidized DJ-1. The H-bonding pattern of Cys106 in the simulations was different in the over-oxidized form compared to the oxidized one. Interactions with the backbone $\mathrm{NH}$ groups of Ala107 and Gly75 in chain B were destabilized (Fig. 3A-D) and in parallel interactions with the backbone NH groups of Gly157 and His126 were formed (Fig. 3I-L). In addition, the H-bond between the backbone oxygen atom of Cys106 and the backbone $\mathrm{NH}$ group of His126 was broken (Fig. S5C-D). At the same time, we observed changes in the backbone $\Psi$ (Fig. 2) and side chain $\chi 1$ angles of Cys106 (Fig. S6). These differences could have important implications as the unusual backbone dihedrals stabilized by strong H-bonds of backbone nitrogen and oxygen atoms of Cys106 have been suggested to play a critical role in the unusually low $\mathrm{p} K_{\mathrm{a}}$ of Cys106 [17]. In line with the exceptionally high reactivity of Cys106 the dihedrals were in the disallowed region of the 
Ramachandran plot during the majority of the simulations. Surprisingly, over-oxidation of Cys106 resulted in higher $\Psi$ values suggesting an increased instability of this residue. Interestingly, the fluctuations in $\chi 1$ of the over-oxidized form in chain B were in contrast to changes of the reduced one, suggesting multiple distinct conformations of the Cys106 side chain. Moreover, the variations in Cys106 $\chi 1$ angle showed correlation with the distances between the side chain sulfur/oxygen atoms of Cys106 and the backbone nitrogen atoms of Ala107 and Gly75 (Fig. 3A-D and Fig. S6).

Besides the different properties of Cys106, further deviations in over-oxidized DJ-1 were observed that mainly involved the C-terminal of chain A and the His126 region of chain B. In particular, less favorable monomer-monomer interactions were detected, and the H-bond between Pro184 and His126 suggested to be critical in dimer formation [54] was completely abolished. Similarly, the SASA of the overoxidized form was slightly larger with major contributions of the C-terminal of chain A and His126 region of chain B suggesting more water exposed hydrophobicity. Furthermore, secondary structure calculations indicated a loss of $\alpha$-helical structure in these two regions. Importantly, these regions did not show any increased fluctuations in the analyzed X-ray structures, indicating that they could be characteristic allosteric consequences of DJ-1 over-oxidation.

Role of the C-terminal. The C-terminal of DJ-1 has been found to be critical for promoting dimer formation and suggested to block higher order assemblies such as hexamers that is the functional form of DJ-1 analogous proteins with protease activity [55]. Moreover, Leu166 in DJ-1 forms a critical interaction with the C-terminal which is impaired in the Leu166Pro disease causing mutation underlining the importance of the C-terminal in the structural integrity of DJ-1 dimers [56]. It has been further suggested that helices 7 and 8 at the $\mathrm{C}$-terminal play a critical role in forming the hydrophobic interface site of
DJ-1, a site for protein-protein interaction [8]. Thus the disruptions found in this region can have serious effects on its functions.

Role of the His126 region. DJ-1 shares significant sequence homology to members of the PfpI protease family (e.g. PH1704, PfpI, ThiJ). Catalytic triad in these enzymes includes a histidine residue that is replaced by an alanine in DJ-1. His126 of DJ-1 was suggested as a replacement for this crucial histidine. However, the distances found between His126, Cys106, Glu18 suggested that DJ-1 is not a functional protease under normal conditions. His 126 forms a $\mathrm{H}$-bond with C-terminal residue Pro184 from the opposite chain that prevents the formation of the catalytic triad. In the simulations of reduced and oxidized DJ-1 the intermonomer His126-Pro184 interaction remained stable. In the over-oxidized form, however, this critical interaction was lost resulting in notable structural changes in the $\mathrm{C}$ terminal of chain A and His126 region of chain B (Fig. S10). After this rearrangement, His126 was able to form H-bond with Cys106 for a short period of time suggesting that the disruption of the His126-Pro184 interaction may lead to structural changes and possibly activation of DJ-1's other functions [51]. It has been also shown that Cterminal truncated forms of DJ-1 have increased protease activity, while wild-type DJ-1 does not. This may be in line with our observations in this study, suggesting a critical role in intermonomer interactions between the C-terminal and His126 region [57]. On the basis of the analysis of DJ-1 crystal structures, Wilson et al. suggested that the formation of over-oxidized Cys106 is sterically disfavored by the surrounding residues, particularly the $\mathrm{C} \beta$ atom of His126 [58]. In accordance with this, we found that the third oxygen of Cys106 initiated structural changes in His126.

DJ-1 over-oxidation causes significant local structural rearrangements. Multiple overoxidized isoforms of DJ-1 have been detected in the brains of patients with idiopathic PD and AD 
[2]. Over-oxidation of Cys106 has been shown to be associated with loss of secondary structure in DJ-1 by CD spectroscopy [26]. However, the CD results in this study did not show significant secondary structural changes in freshly prepared over-oxidized DJ-1 compared to oxidized or reduced DJ-1. In the simulations over-oxidized DJ-1 already showed characteristic differences and loss of secondary structure at the local level, however these were not yet significant at the full protein level. Further, the higher water exposed surface area and loss of intermonomer interactions found in the simulation of over-oxidized DJ-1 also suggest the dimeric state of over-oxidized DJ-1 may be less stable compared to reduced and oxidized forms.

Structural characteristics of the Cys106 containing pocket. Due to the lack of experimental structures of apo DJ-1 in different oxidation states, MD simulations were carried out to identify differences that could be exploited for structure-based drug design of oxidation-state specific DJ-1 ligands. As a buried, nucleophilic residue Cys106 provides a particularly interesting targetable site for drug discovery. On the basis of our simulations the over-oxidized DJ-1 had a larger binding site around Cys106. A new cavity in the direction of His126 was observed between the Cys106 binding site and the hydrophobic interface site (Fig. 6) [14]. Since this cavity could not be detected in either reduced or oxidized DJ-1, we suggest that it may be selectively targeted by small molecule ligands.

\section{CONCLUSIONS}

To conclude, this study presents the first comprehensive computational structural and biophysical characterization of physiologically relevant oxidation states of DJ-1 protein. According to our findings, the overall structure and dynamics of DJ-1 in different oxidation states were similar globally, while the local differences may have implications on its stability, function and its link to PD and other diseases. In particular, reduced DJ-1 lacked some critical local interactions when compared to oxidized and overoxidized DJ-1 due to lack of side chain oxygens in Cys106. In addition, the characteristic interaction between Cys106 and the protonated Glu18 was much weaker in reduced DJ-1. Local structural differences in the Cys106 containing pocket between the various oxidation states of DJ-1 were identified, which may be responsible for effects on its suggested functions. The third oxygen in the over-oxidized DJ-1 resulted in a partial loss of helical secondary structure at the C-terminal and His126 region, reduced strength of the monomermonomer interactions and increased solvent exposed surface area compared to oxidized and reduced states of DJ-1. Taken together, we speculate that over-oxidation may further trigger loss of function due to the structural destabilization of the dimeric state as well as the active conformation of DJ-1. In a number of neurodegenerative diseases such as in Parkinson's disease, high oxidative stress critically contributes to the onset and progression of the disease. High oxidative stress inducing over-oxidization of DJ-1 can lead to loss of function potentially by local structural modification and by the destabilization of DJ-1 dimeric structure. Such loss of DJ-1 function would result in reduction of redox protein homeostasis in dopaminergic neurons and thus the increased progression of the disease. Moreover, the distinct Cys 106 pocket characteristics of overoxidized DJ-1 may provide a basis for identifying oxidation-state selective ligands. These may have applicability as imaging agents for the detection and characterization of over-oxidized DJ-1 in a number of diseases. 


\section{AUTHOR CONTRIBUTIONS}

G.T. conceived and coordinated the study and analyzed all data. R.K. and M.Z. designed, performed and analyzed all simulations. B.J. performed AMBER parameterization for oxidized cysteine residues and MM/GBSA calculations. R.K. designed, performed and analyzed the LC-MS experiments. K.S. and B.F. designed, performed and analyzed the DLS experiments. A.C. and E.N. designed, performed and analyzed the DSC experiments shown in Fig. 7A. F.Z. and T.B. designed, performed and analyzed the CD experiments shown in Figure 7B. R.K., G.T. and M.Z. wrote the paper. All authors reviewed the results and approved the final version of the manuscript.

\section{REFERENCES}

[1] Ariga, H., Takahashi-Niki, K., Kato, I., Maita, H., Niki, T., and Iguchi-Ariga, S. (2013) Neuroprotective Function of DJ-1 in Parkinson's Disease. Oxidative Medicine and Cellular Longevity 2013, 1-9.

[2] Choi, J., Sullards, M. C., Olzmann, J. A., Rees, H. D., Weintraub, S. T., Bostwick, D. E., . . Li, L. (2006) Oxidative Damage of DJ-1 Is Linked to Sporadic Parkinson and Alzheimer Diseases. Journal of Biological Chemistry, 281(16), 10816-10824.

[3] Kahle, P. J., Waak, J., \& Gasser, T. (2009) DJ-1 and prevention of oxidative stress in Parkinson's disease and other age-related disorders. Free Radical Biology and Medicine, 47(10), 1354-1361.

[4] Aleyasin, H., Rousseaux, M. W., Phillips, M., Kim, R. H., Bland, R. J., Callaghan, S., ... Park, D. S. (2007) The Parkinson's disease gene DJ-1 is also a key regulator of stroke-induced damage. Proceedings of the National Academy of Sciences, 104(47), 18748-18753.

[5] Inberg, A., \& Linial, M. (2010) Protection of Pancreatic B-Cells from Various Stress Conditions Is Mediated by DJ-1. Journal of Biological Chemistry, 285(33), 25686-25698.

[6] Jain, D., Jain, R., Eberhard, D., Eglinger, J., Bugliani, M., Piemonti, L., ... Lammert, E. (2012) Ageand diet-dependent requirement of DJ-1 for glucose homeostasis in mice with implications for human type 2 diabetes. Journal of Molecular Cell Biology, 4(4), 221-230.

[7] Cao, J., Lou, S., Ying, M., \& Yang, B. (2015) DJ-1 as a human oncogene and potential therapeutic target. Biochemical Pharmacology, 93(3), 241-250.

[8] Bonifati, V., Rizzu, P., van Baren, M. J., Schaap, O., Breedveld, G. J., Krieger, E., ... Heutink, P. (2002) Mutations in the DJ-1 gene associated with autosomal recessive early-onset parkinsonism. Science, 5604, 256-259. 

CNS. Brain research, 1, 102-111.

[10] Taira, T., Saito, Y., Niki, T., Iguchi-Ariga, S. M., Takahashi, K., \& Ariga, H. (2004) DJ-1 has a role in antioxidative stress to prevent cell death. EMBO reports, 5(2), 213-218.

[11] Meulener, M. C., Graves, C. L., Sampathu, D. M., Armstrong-Gold, C. E., Bonini, N. M., \& Giasson, B. I. (2005) DJ-1 is present in a large molecular complex in human brain tissue and interacts with $\alpha$-synuclein. Journal of Neurochemistry, 93(6), 1524-1532.

[12] Shendelman, S., Jonason, A., Martinat, C., Leete, T., \& Abeliovich, A. (2004) DJ-1 Is a RedoxDependent Molecular Chaperone That Inhibits $\alpha$-Synuclein Aggregate Formation. PLoS Biology, 2(11).

[13] Chen, J., Li, L., \& Chin, L. (2010) Parkinson disease protein DJ-1 converts from a zymogen to a protease by carboxyl-terminal cleavage. Human Molecular Genetics, 19(12), 2395-2408.

[14] Lee, S., Kim, S. J., Kim, I., Ko, J., Jeong, C., Kim, G., . . . Cha, S. (2003) Crystal Structures of Human DJ-1 and Escherichia coli Hsp31, Which Share an Evolutionarily Conserved Domain. Journal of Biological Chemistry, 278(45), 44552-44559.

[15] Cha, S., Jung, H. I., Jeon, H., An, Y. J., Kim, I., Yun, S., . . Kang, S. (2008) Crystal Structure of Filamentous Aggregates of Human DJ-1 Formed in an Inorganic Phosphate-dependent Manner. Journal of Biological Chemistry, 283(49), 34069-34075.

[16] Saito, Y., Akazawa-Ogawa, Y., Matsumura, A., Saigoh, K., Itoh, S., Sutou, K., ... Noguchi, N. (2016) Oxidation and interaction of DJ-1 with $20 \mathrm{~S}$ proteasome in the erythrocytes of early stage Parkinson's disease patients. Scientific Reports, 6, 30793.

[17] Witt, A. C., Lakshminarasimhan, M., Remington, B. C., Hasim, S., Pozharski, E., \& Wilson, M. A. (2008) Cysteine pKa depression by a protonated glutamic acid in human DJ-1. Biochemistry, 28, 74307440.

[18] Madzelan, P., Labunska, T., \& Wilson, M. A. (2012) Influence of peptide dipoles and hydrogen bonds on reactive cysteine $\mathrm{pKa}$ values in fission yeast DJ-1. The FEBS journal, 22, 4111-4120.

[19] Mitsumoto, A., Nakagawa, Y., Takeuchi, A., Okawa, K., Iwamatsu, A., \& Takanezawa, Y. (2001) Oxidized forms of peroxiredoxins and DJ-1 on two-dimensional gels increased in response to sublethal levels of paraquat. Free radical research, 3, 301-310.

[20] Kinumi, T., Kimata, J., Taira, T., Ariga, H., and Niki, E. (2004) Cysteine-106 of DJ-1 is the most sensitive cysteine residue to hydrogen peroxide-mediated oxidation in vivo in human umbilical vein endothelial cells. Biochemical and Biophysical Research Communications 317, 722-728.

[21] Canet-Avilés, R. M., Wilson, M. A., Miller, D. W., Ahmad, R., McLendon, C., Bandyopadhyay, ... Cookson, M. R. (2004) The Parkinson's disease protein DJ-1 is neuroprotective due to cysteine-sulfinic acid-driven mitochondrial localization. Proceedings of the National Academy of Sciences of the United States of America, 24, 9103-9108.

[22] Rizzu, P., Hinkle, D., Zhukareva, V., Bonifati, V., Severijnen, L., Martinez, D., ... Heutink, P. (2003) DJ-1 colocalizes with tau inclusions: A link between parkinsonism and dementia. Annals of Neurology 55, 113-118.

[23] Bandopadhyay, R. (2004) The expression of DJ-1 (PARK7) in normal human CNS and idiopathic Parkinson's disease. Brain 127, 420-430.

[24] Giasson, B. (2000) Oxidative Damage Linked to Neurodegeneration by Selective alpha -Synuclein Nitration in Synucleinopathy Lesions. Science 290, 985-989.

[25] Hartmann, A. (2004) Postmortem studies in Parkinson's disease. Dialogues in Clinical Neuroscience, 6(3), 281-293. 

regulates its chaperone activity toward alpha-synuclein. Journal of molecular biology, 4, 1036-1048.

[27] Premkumar, L., Dobaczewska, M. K., \& Riedl, S. J. (2011) Identification of an artificial peptide motif that binds and stabilizes reduced human DJ-1. Journal of structural biology, 3, 414-418.

[28] Herrera, F. E., Zucchelli, S., Jezierska, A., Lavina, Z. S., Gustincich, S., \& Carloni, P. (2007) On the Oligomeric State of DJ-1 Protein and Its Mutants Associated with Parkinson Disease: A Combined Computational and in vitro Study. Journal of Biological Chemistry, 282(34), 24905-24914.

[29] Anderson, P. C., \& Daggett, V. (2008) Molecular basis for the structural instability of human DJ-1 induced by the L166P mutation associated with Parkinson's disease. Biochemistry, 36, 9380-9393. [30] McLachlan, A.D., (1982) Rapid Comparison of Protein Structures. Acta Cryst, A38, 871-873. [31] Homeyer, N., Horn, A. H. C., Lanig, H., and Sticht, H. (2006) AMBER force-field parameters for phosphorylated amino acids in different protonation states: phosphoserine, phosphothreonine, phosphotyrosine, and phosphohistidine. J. Mol. Model. 12, 281-289.

[32] Frisch, M. J., Trucks, G. W., Schlegel, H. B., Scuseria, G. E., Robb, M. A., Cheeseman, J. R., ... Fox, D. J. (2009) Gaussian 09, Revision D.01 Gaussian, Inc., Wallingford CT.

[33] Case, D. A., Babin, V., Berryman, J. T., Betz, R. M., Cai, Q., Cerutti, D. S., ... Kollman, P. A. (2014) AMBER 14, University of California, San Francisco.

[34] Dupradeau, F.-Y., Pigache, A., Zaffran, T., Savineau, C., Lelong, R., Grivel, N., Lelong, D., Rosanski, W., and Cieplak, P. (2010) The R.E.D. tools: advances in RESP and ESP charge derivation and force field library building. Phys. Chem. Chem. Phys. 12, 7821-7839.

[35] Wang, J., Wolf, R. M., Caldwell, J. W., Kollman, P. A., and Case, D. A. (2004) Development and testing of a general amber force field. J. Comput. Chem. 25, 1157-1174.

[36] Wang, J., Wang, W., Kollman, P. A., and Case, D. A. (2006) Automatic atom type and bond type perception in molecular mechanical calculations. J. Mol. Graph. Model. 25, 247-60.

[37] Lindorff-Larsen, K., Piana, S., Palmo, K., Maragakis, P., Klepeis, J. L., Dror, R. O., \& Shaw, D. E. (2010) Improved side-chain torsion potentials for the Amber ff99SB protein force field. Proteins, 8, $1950-1958$.

[38] Pronk, S., Páll, S., Schulz, R., Larsson, P., Bjelkmar, P., Apostolov, R., ... \& Hess, B. (2013) GROMACS 4.5: a high-throughput and highly parallel open source molecular simulation toolkit. Bioinformatics, 29(7).

[39] Hornak, V., Abel, R., Okur, A., Strockbine, B., and Roitberg, A. (2006) Comparison of multiple Amber force fields and development of improved protein backbone parameters. Proteins 65, 712-725.

[40] Miller, B. R., Mcgee, T. D., Swails, J. M., Homeyer, N., Gohlke, H., \& Roitberg, A. E. (2012). MMPBSA.py: An Efficient Program for End-State Free Energy Calculations. Journal of Chemical Theory and Computation, 8(9), 3314-3321.

[41] Onufriev, A.; Bashford, D.; Case, D.A. Onufriev, A., Bashford, D., \& Case, D. A. (2004) Exploring protein native states and large-scale conformational changes with a modified generalized born model. Proteins, 2, 383-394.

[42] Weiser, J., Shenkin, P., and Still, W. (1999) Approximate atomic surfaces from linear combinations of pairwise overlaps (LCPO). J. Comput. Chem. 20, 217-230.

[43] Zhang, Z., \& Marshall, A. G. (1998) A universal algorithm for fast and automated charge state deconvolution of electrospray mass-to-charge ratio spectra. Journal of the American Society for Mass Spectrometry, 9(3), 225-233. 
[44] Andrade, M., Chacón, P., Merelo, J., \& Morán, F. (1993) Evaluation of secondary structure of proteins from UV circular dichroism spectra using an unsupervised learning neural network. "Protein Engineering, Design and Selection", 6(4), 383-390.

[45] Ortega, A., Amorós, D., and García de la Torre, J. (2011) Prediction of Hydrodynamic and Other Solution Properties of Rigid Proteins from Atomic- and Residue-Level Models. Biophysical Journal, 101, 892-898.

[46] Defelipe, L., Lanzarotti, E., Gauto, D., Marti, M., and Turjanski, A. (2015) Protein Topology Determines Cysteine Oxidation Fate: The Case of Sulfenyl Amide Formation among Protein Families. PLOS Computational Biology 11, e1004051.

[47] Wilson, M. A., Ringe, D., Petsko, G. A. (2005) The atomic resolution crystal structure of the YajL (ThiJ) protein from Escherichia coli: a close prokaryotic homologue of the Parkinsonism-associated protein DJ-1. J. Mol. Biol. 353(3), 678-691.

[48] Nagahara, N. (2011) Catalytic Site Cysteines of Thiol Enzyme: Sulfurtransferases. Journal of Amino Acids 2011, 1-7.

[49] van Montfort, R. L., Congreve, M., Tisi, D., Carr, R., \& Jhoti, H. (2003) Oxidation state of the active-site cysteine in protein tyrosine phosphatase 1B. Nature, 6941, 773-777.

[50] Lakshminarasimhan, M., Maldonado, M. T., Zhou, W., Fink, A. L., \& Wilson, M. A. (2008) Structural Impact of Three Parkinsonism-Associated Missense Mutations on Human DJ-1. Biochemistry, 47(5), 1381-1392.

[51] Lin J, Prahlad J, Wilson M. A. (2012) Conservation of oxidative protein stabilization in an insect homologue of parkinsonism-associated protein DJ-1. Biochemistry. 2012;51(18):3799-807.

[52] Görner K., Holtorf E., Odoy S., Nuscher B., Yamamoto A., Regula J. T., ... Kahle P. J. (2004) Differential effects of Parkinson's disease-associated mutations on stability and folding of DJ-1. J. Biol. Chem. (279) 6943-6951.

[53] Pakavathkumar, P., Sharma, G., Kaushal, V., Foveau, B., \& Leblanc, A. C. (2015) Methylene blue inhibits caspases by oxidation of the catalytic cysteine. Alzheimer's \& Dementia, 11(7).

[54] Honbou, K., Suzuki, N. N., Horiuchi, M., Niki, T., Taira, T., Ariga, H., \& Inagaki, F. (2003) The crystal structure of DJ-1, a protein related to male fertility and Parkinson's disease. J Biol Chem, 33, 31380-31384.

[55] Huai, Q., Sun, Y., Wang, H., Chin, L.S., Li, L., Robinson, H., Ke, H. (2003) Crystal structure of DJ1/RS and implication on familial Parkinson's disease. FEBS Lett. 549, 171-175.

[56] Tao, X., \& Tong, L. (2003) Crystal structure of human DJ-1, a protein associated with early onset Parkinson's disease. The Journal of biological chemistry, 33, 31372-31379.

[57] Mitsugi, H., Niki, T., Takahashi-Niki, K., Tanimura, K., Yoshizawa-Kumagaye, K., Tsunemi, M., . . . Ariga, H. (2013) Identification of the recognition sequence and target proteins for DJ-1 protease. FEBS Letters, 587(16), 2493-2499.

[58] Wilson, M. A. (2011) The Role of Cysteine Oxidation in DJ-1 Function and Dysfunction. Antioxidants \& Redox Signaling, 15(1), 111-122. 


\section{FOOTNOTES}

None

\section{FIGURE LEGENDS}

Figure 1. (A) DJ-1 homodimer with Cys106 highlighted. (B) Geometry of reduced (CYM), oxidized (CSD) and over-oxidized (OCS) states of cysteine. Atoms are colored as follows: $\mathrm{C}$ (green), $\mathrm{O}$ (red), $\mathrm{N}$ (blue), S (yellow). Per residue average C $\alpha$ RMSD (C) of the analyzed X-ray structures of DJ-1 (blue) (+/S.D. values are indicated in black). Per residue secondary structure propensity (D) of the analyzed X-ray structures of DJ-1: $\alpha$-helical (blue) and beta-sheet (red) structures are indicated.

Figure 2. Backbone $\Phi$ and $\Psi$ dihedral angle distributions for chain $A(A, C)$ and chain $B(B, D)$ for the reduced, oxidized and over-oxidized DJ-1. Angles calculated from the available X-ray structures are shown as blue triangles (A, B).

Figure 3. Time series of interatomic distances and atomic representations for reduced (gray), oxidized (red) and over-oxidized (green) DJ-1. Data for Chain A and B are displayed on the left and right, respectively. For distances involving Cys106: the closest distance from either the side chain S (reduced DJ-1) or any of the side chain Os (oxidized or over-oxidized DJ-1) is shown. Line values are moving averages with a 2ns window. A-B. Cys106 to backbone N atom of Ala107. C-D. Cys106 to backbone N atom of Gly75. E-F. Cys106 to the protonated side chain O atom of Glu18. G-H. Protonated side chain $\mathrm{O}$ atom of Glu18 to the side chain O atom of Glu15. I-J. Cys106 to backbone N atom of Gly157. K-L. Cys106 to side chain NE2 atom of His126.

Figure 4. DJ-1 monomers separated showing largest intermonomer interaction energy differences (A, B) and largest solvent accessible surface area (SASA) differences (C, D) between reduced and over-oxidized $(\mathrm{A}, \mathrm{C})$ or oxidized and over-oxidized $(\mathrm{B}, \mathrm{D})$ states. Blue indicates weaker intermonomer interactions and more SASA in over-oxidized state, while red indicates stronger intermonomer interaction and less SASA in over-oxidized state.

Figure 5. Helical (A, B) and unstructured ("loop") (C, D) secondary structure content of the C-terminal of chain A (A, C) and the His126 region in chain B (B, D) of the reduced (grey), oxidized (red) and overoxidized (green) DJ-1 systems calculated by DSSP.

Figure 6. Cys106 binding site of reduced (CYM), oxidized (CSD) and over-oxidized (OCS) DJ-1 in the second monomer (chain B). Access to the binding site is indicated by black arrows.

Figure 7. (A) Thermodynamic stability of reduced (grey), oxidized (red) and over-oxidized (green) DJ-1 protein determined by DSC. Determined $\mathrm{T}_{\mathrm{m}}$ were $62.2,75.0$ and $75.1{ }^{\circ} \mathrm{C}$, respectively, for reduced, oxididzed and over-oxidized DJ-1. (B) CD spectra of the reduced (44 $\mu \mathrm{M}$, grey), oxidized (40 $\mu \mathrm{M}$, red), and over-oxidized ( $46 \mu \mathrm{M}$, green) form of DJ-1 in the far-UV region. The results of secondary structure analysis performed using the K2d program for all three forms of DJ-1 are: $\alpha$-helix (37\%), $\beta$-sheet (26\%), and random coil (38\%). 


\section{TABLE LEGENDS}

Table 1. Geometric data extracted from experimental protein structures and calculated by GAFF for the modified cysteine residues: 3 -sulfinoalanine (CSD) and cysteine sulfonic acid (OCS).

Table 2. Calculated charges for the modified cysteine residues: 3-sulfinoalanine (CSD) and cysteine sulfonic acid (OCS).

Table 3. Hydrodynamic diameter derived from DLS experiments and calculated for X-ray structures and from MD simulations of DJ-1. *: the oxidation state of DJ-1 in the X-ray structures is ambiguous [27], therefore the average value of all X-ray structures is indicated. 


\section{FIGURES}

Figure 1.

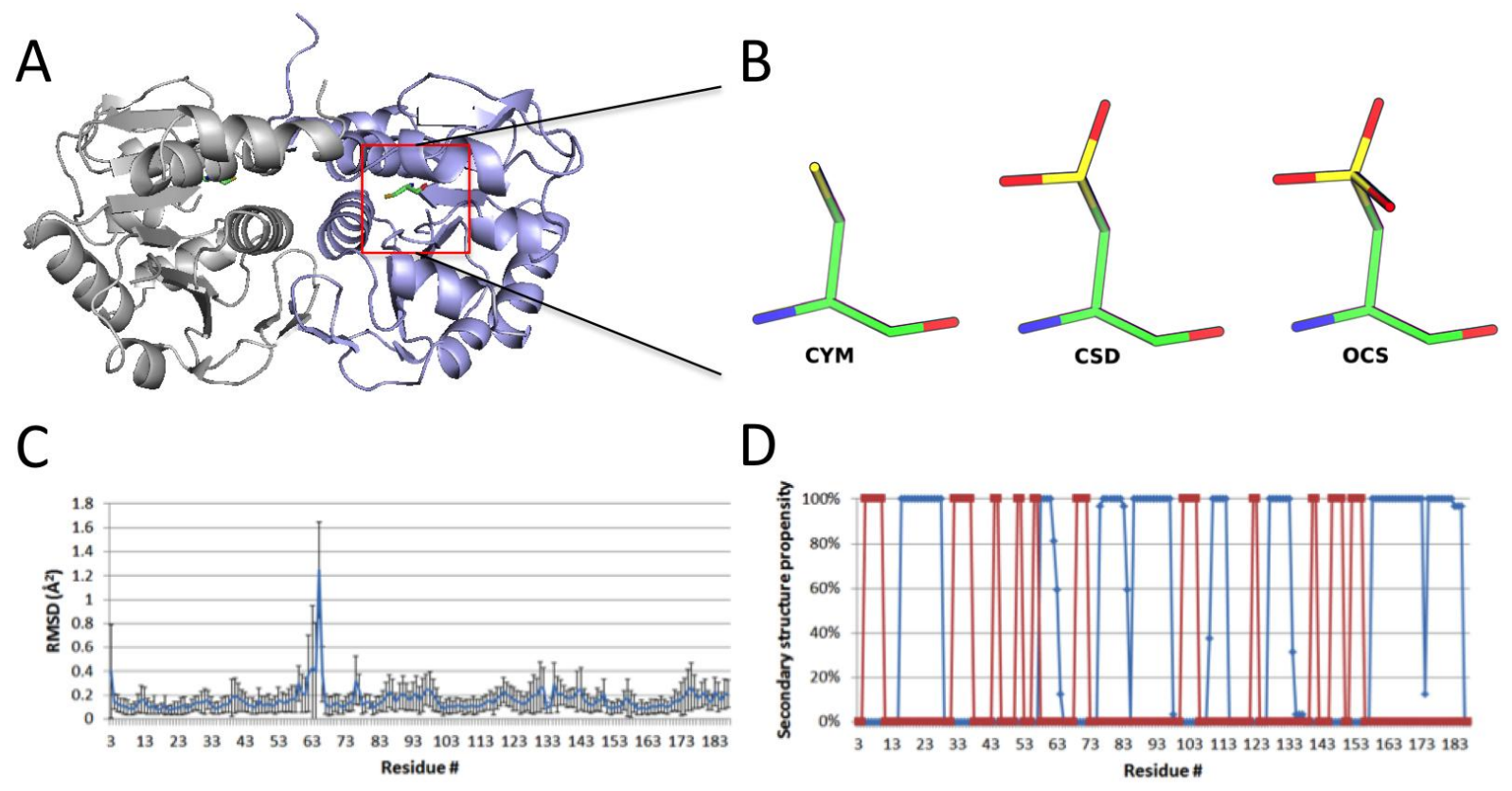

Figure 2.
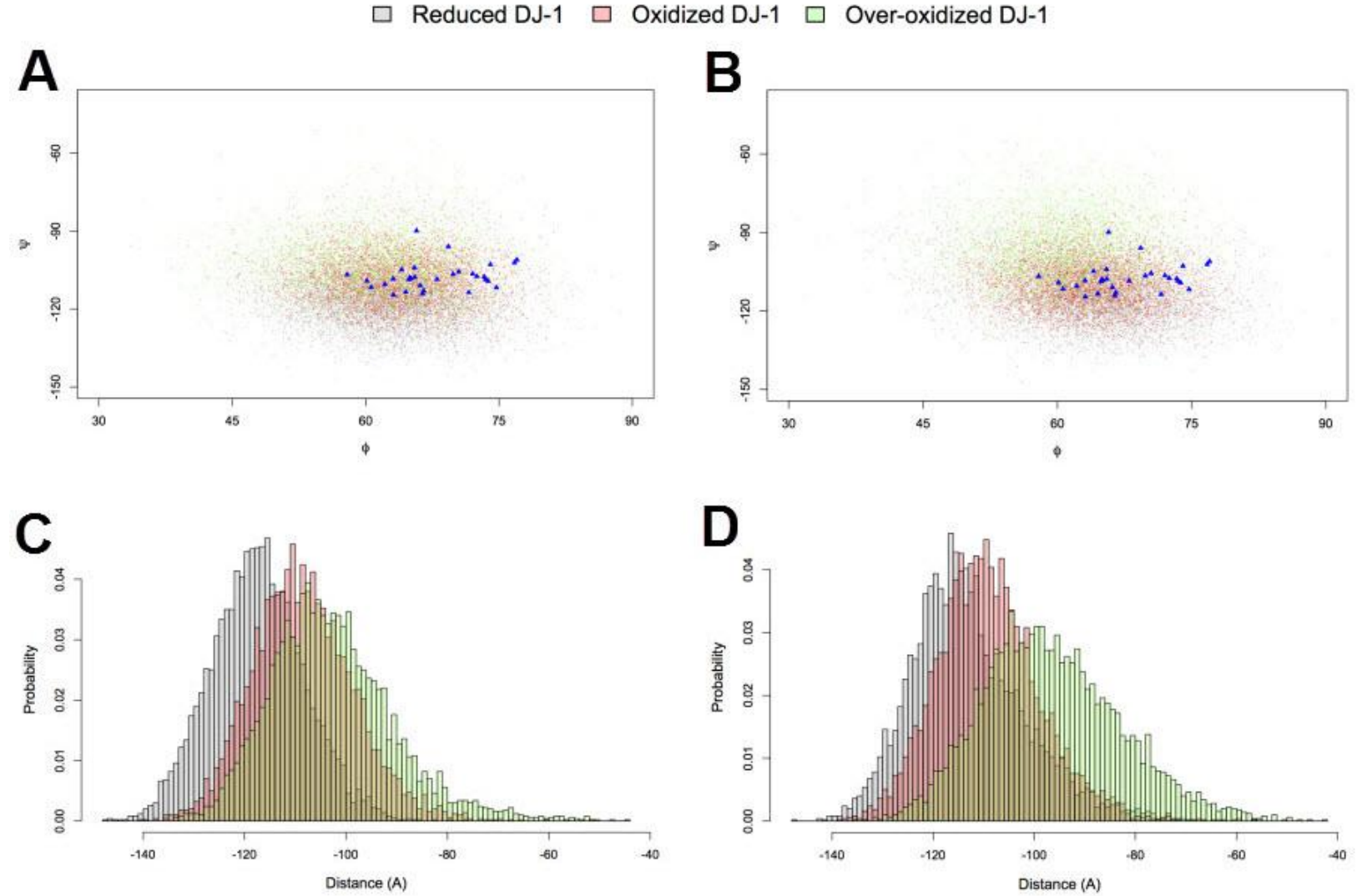
Figure 3.
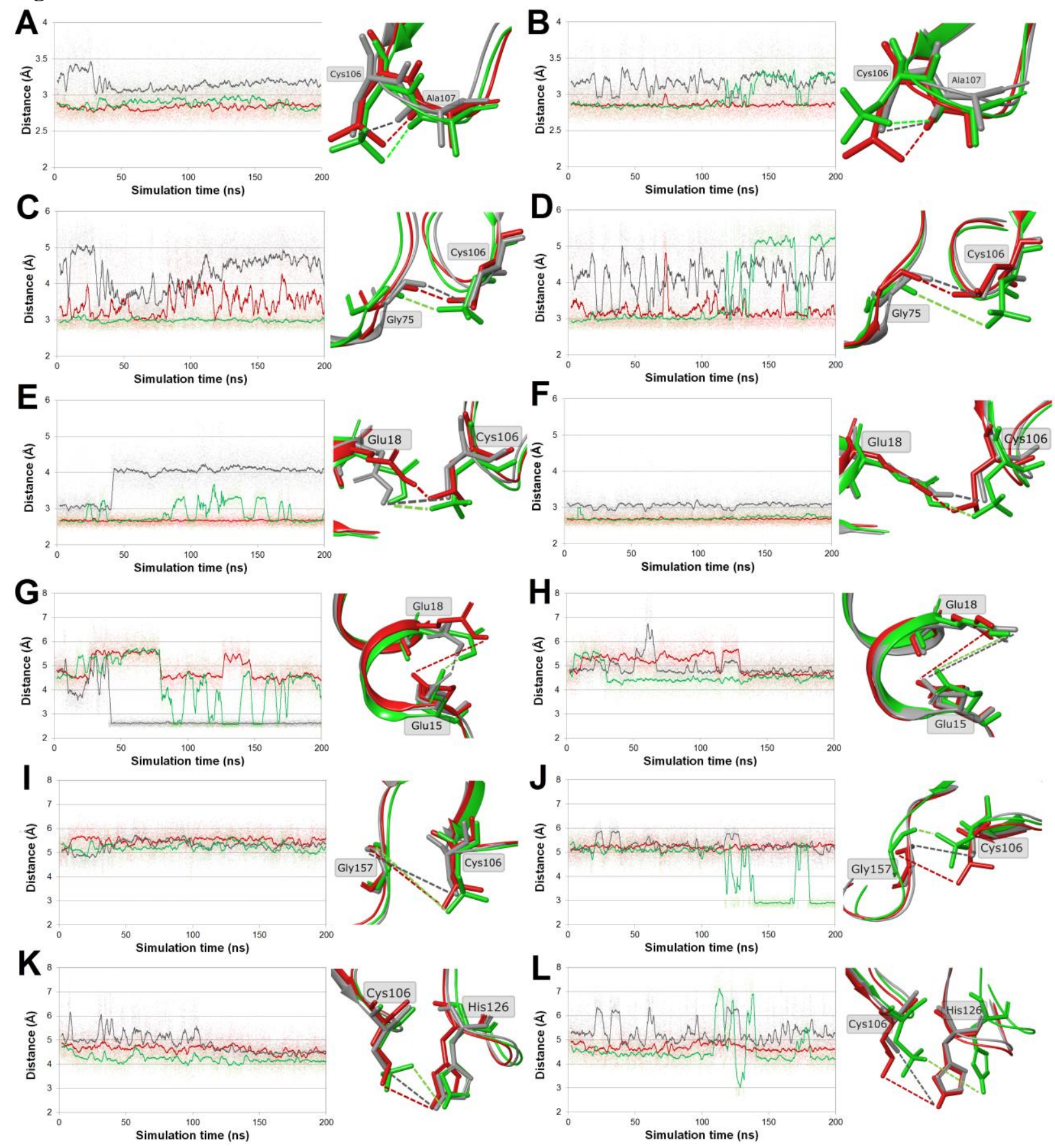
Figure 4.
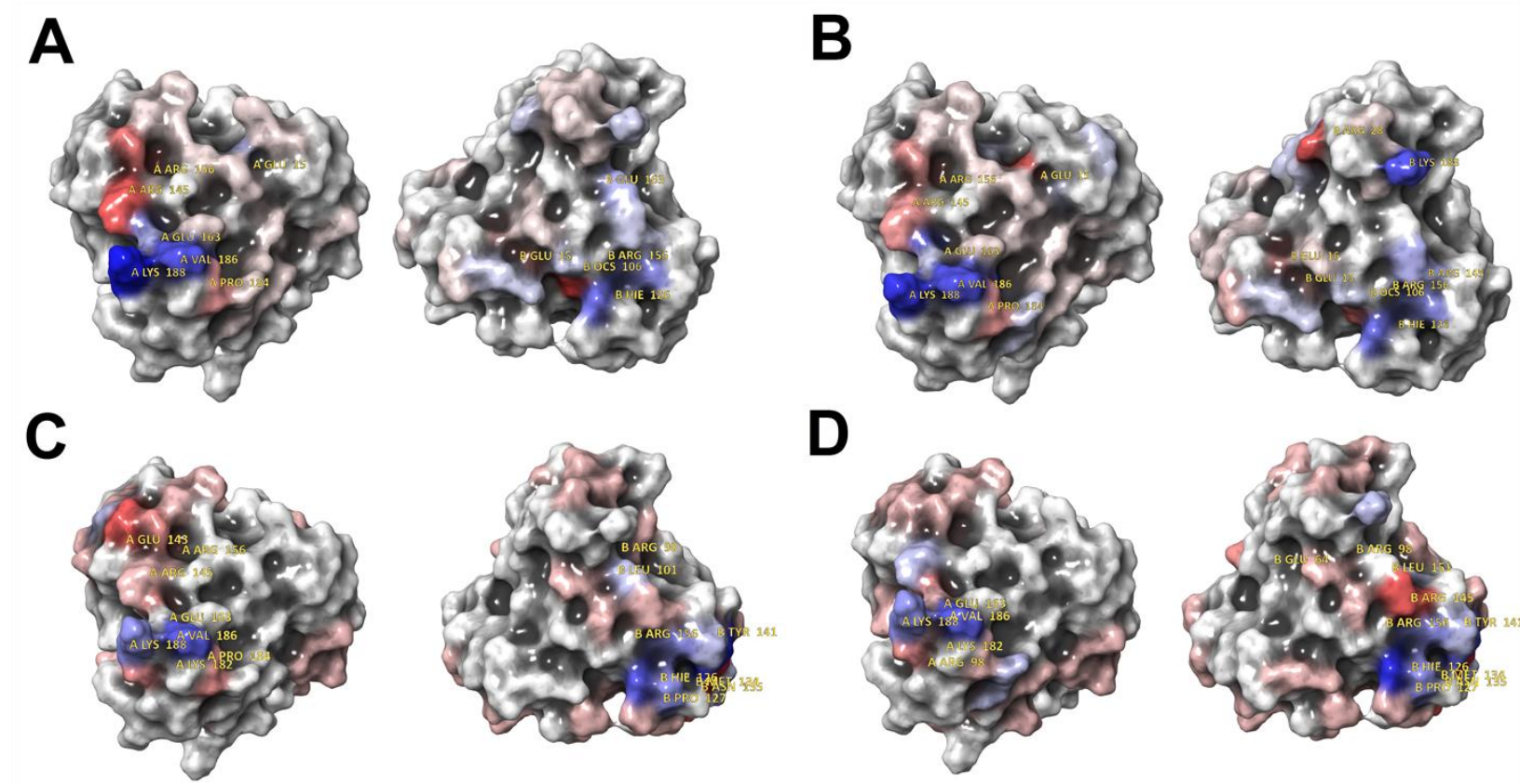

D
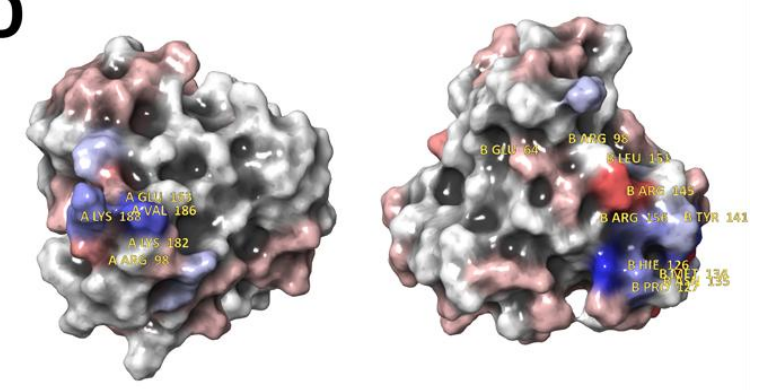

Figure 5.
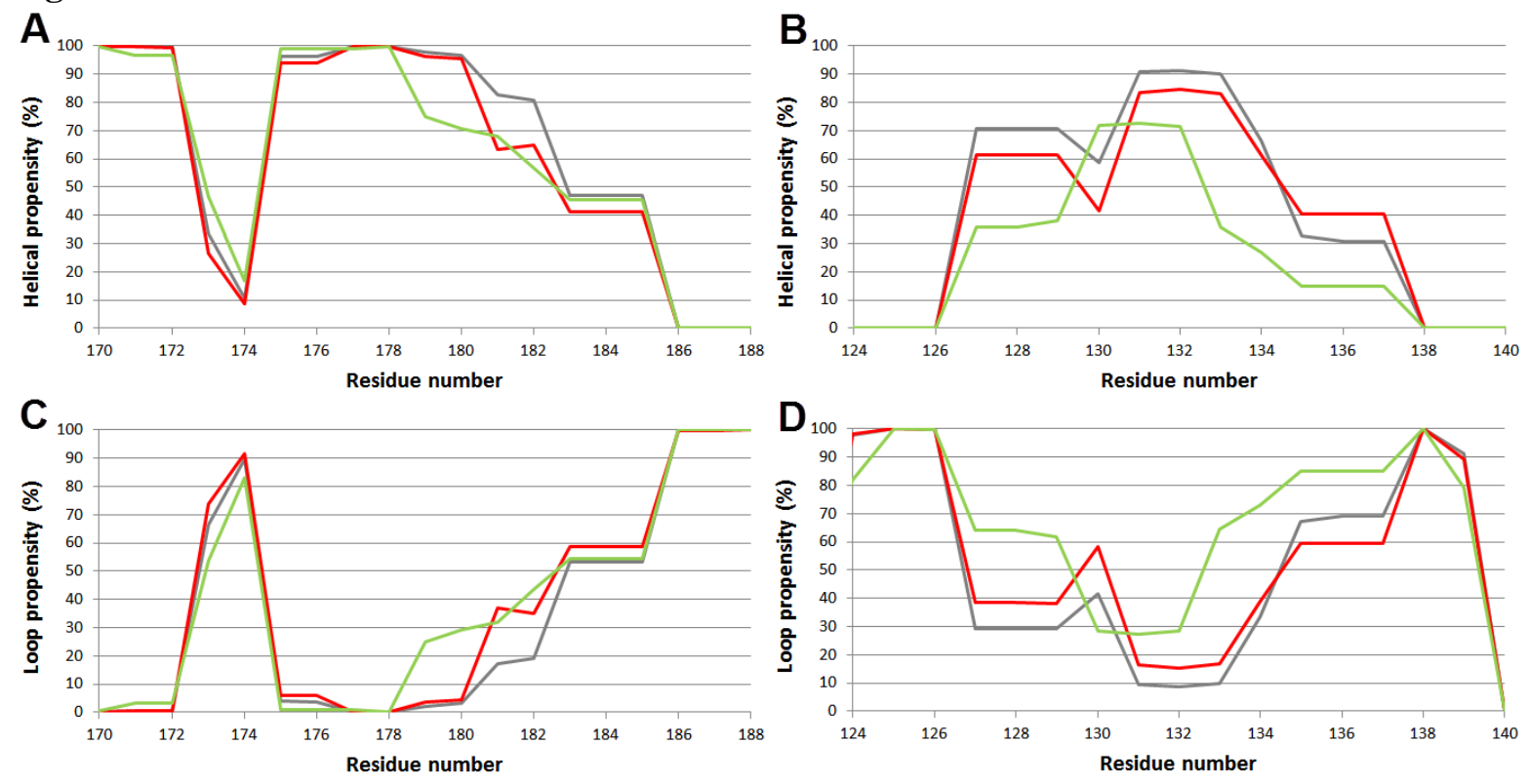

Figure 6.
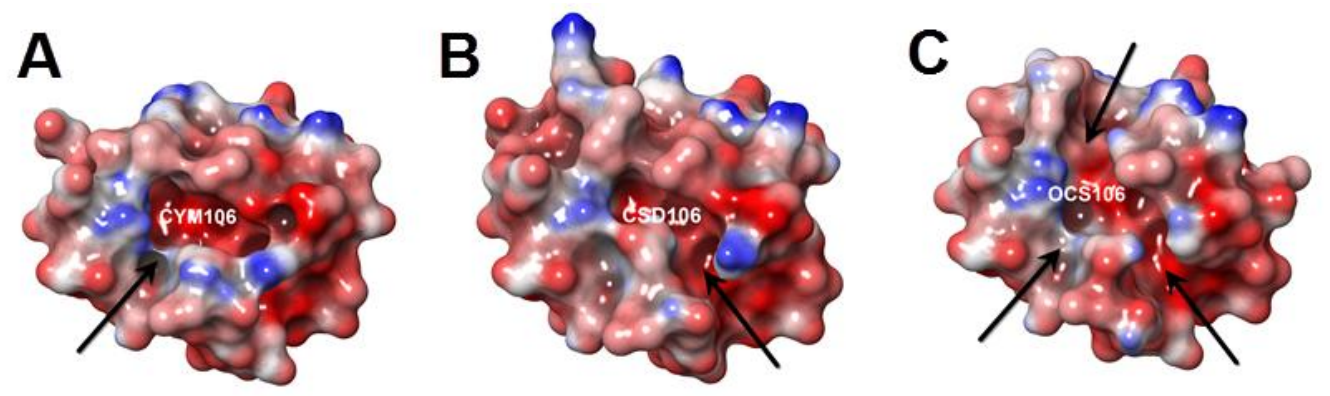
Figure 7.
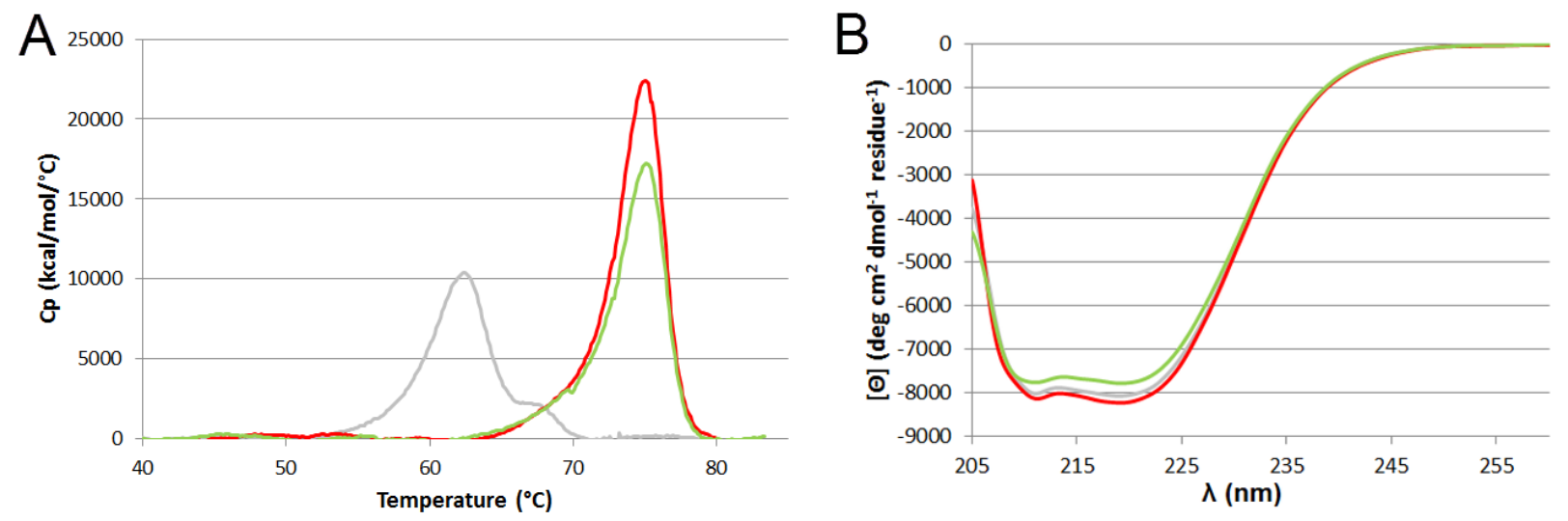

TABLES

Table 1.

\begin{tabular}{|c|c|c|}
\hline & Experimental & GAFF \\
\hline \multicolumn{3}{|l|}{ CSD } \\
\hline $\mathrm{d}(\mathrm{CB}-\mathrm{SG}) / \AA$ & $1.776 \pm 0.066$ & 1.807 \\
\hline $\mathrm{d}(\mathrm{SG}-\mathrm{ODx}) / \AA$ & $1.417 \pm 0.068$ & 1.497 \\
\hline$\alpha(\mathrm{CA}-\mathrm{CB}-\mathrm{SG}) /^{\circ}$ & $115.831 \pm 2.735$ & 110.070 \\
\hline$\alpha(\mathrm{CB}-\mathrm{SG}-\mathrm{ODx}) /^{\circ}$ & $108.285 \pm 9.637$ & 106.210 \\
\hline$\alpha(\mathrm{OD} 1-\mathrm{SG}-\mathrm{OD} 2) /^{\circ}$ & $110.583 \pm 7.556$ & 110.610 \\
\hline \multicolumn{3}{|l|}{ OCS } \\
\hline $\mathrm{d}(\mathrm{CB}-\mathrm{SG}) / \AA$ & $1.760 \pm 0.064$ & 1.774 \\
\hline $\mathrm{d}(\mathrm{SG}-\mathrm{ODx}) / \AA$ & $1.451 \pm 0.080$ & 1.436 \\
\hline$\alpha(\mathrm{CA}-\mathrm{CB}-\mathrm{SG}) /^{\circ}$ & $116.290 \pm 3.494$ & 110.000 \\
\hline$\alpha(\mathrm{CB}-\mathrm{SG}-\mathrm{ODx}) /^{\circ}$ & $107.353 \pm 4.529$ & 108.320 \\
\hline$\alpha(\mathrm{ODx}-\mathrm{SG}-\mathrm{ODy}) /^{\circ}$ & $111.214 \pm 4.767$ & 119.730 \\
\hline
\end{tabular}


Table 2.

\begin{tabular}{l|l|l} 
Atom $\backslash$ Residue & CSD & OCS \\
\hline CA (HA) & $0.0950(-0.1390)$ & $-0.2186(0.1735)$ \\
\hline CB (HB) & $-0.0064(0.0352)$ & $0.0188(0.0176)$ \\
\hline SG & 0.3128 & 1.1326 \\
\hline ODx & -0.6092 & -0.6757
\end{tabular}

Table 3.

\begin{tabular}{l|l|l|l} 
& Exp. Dh $(\mathrm{nm}) \pm \mathrm{SD}$ & $\begin{array}{l}\text { X-ray Dh }(\mathrm{nm}) \pm \\
\text { SD }\end{array}$ & $\begin{array}{l}\text { Simulation Dh } \\
(\mathrm{nm}) \pm \mathrm{SD}\end{array}$ \\
\cline { 1 - 1 } Reduced & $5.361 \pm 0.182$ & \multirow{3}{*}{$5.540 \pm 0.056^{*}$} & $5.604 \pm 0.022$ \\
\cline { 1 - 2 } Oxidized & $5.402 \pm 0.140$ & & $5.604 \pm 0.022$ \\
\cline { 1 - 2 } Over-oxidized & $5.610 \pm 0.127$ & & $5.614 \pm 0.022$
\end{tabular}




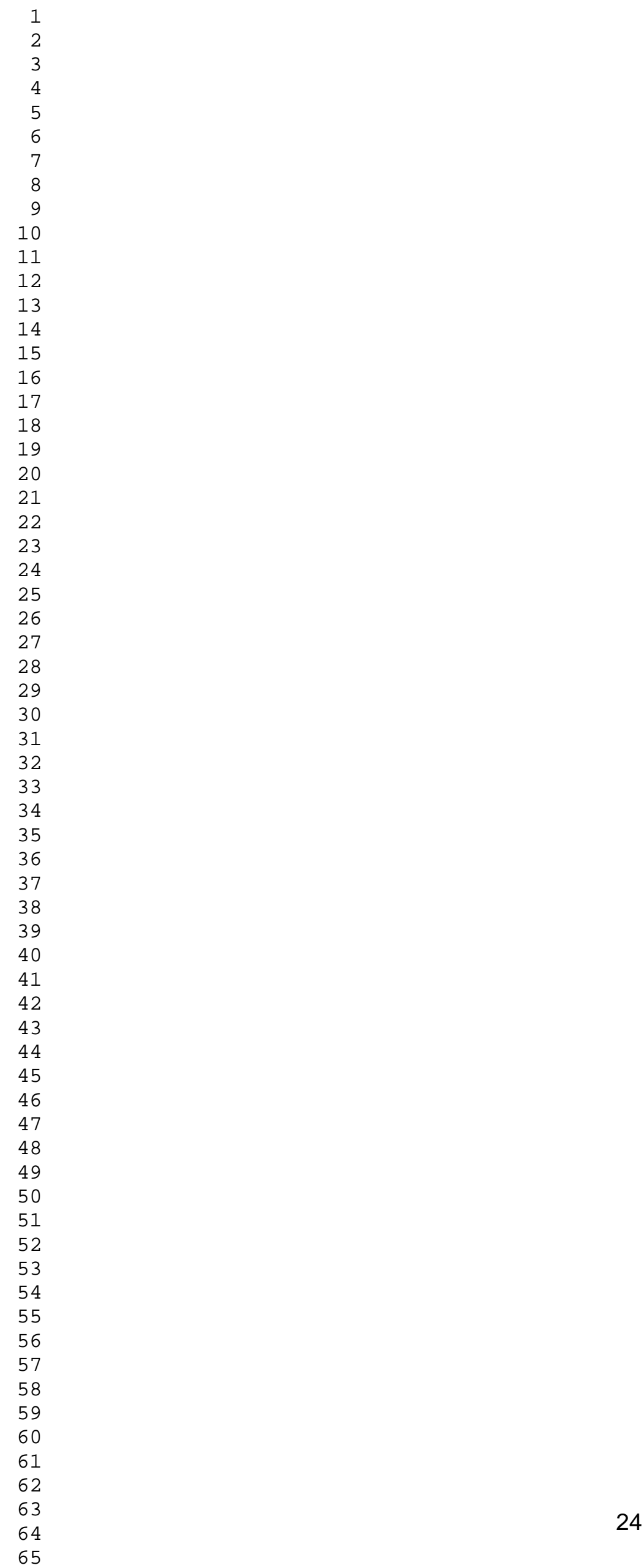




\section{SUPPLEMENTAL DATA}

\section{Structural Features of Human DJ-1 in Distinct Cys106 Oxidative States and their Relevance to its Loss of Function in Disease}

Róbert Kiss ${ }^{1, *}$, Max Zhu ${ }^{2, *}$, Balázs Jójárt ${ }^{3}$, András Czajlik ${ }^{1}$, Katalin Solti ${ }^{1}$, Balázs Fórizs ${ }^{2}$, Éva Nagy ${ }^{1}$, Ferenc Zsila ${ }^{4}$, Tamás Beke-Somfai ${ }^{4}$, Gergely Tóth ${ }^{1,2}$

${ }^{1}$ MTA-TTK-NAP B - Drug Discovery Research Group - Neurodegenerative Diseases, Institute of Organic Chemistry, Research Center for Natural Sciences, Hungarian Academy of Sciences, Budapest, Hungary

${ }^{2}$ Cantabio Pharmaceuticals, Sunnyvale, California, USA

${ }^{3}$ Department of Chemical Informatics, Faculty of Education, University of Szeged, Boldogasszony sgt. 6 , Szeged 6725, Hungary

${ }^{4}$ Biomolecular Self-Assembly Group, Institute of Materials and Environmental Chemistry, Research Centre for Natural Sciences, Hungarian Academy of Sciences, Budapest, Hungary

* Both authors contributed equally to this manuscript.

Running Title: Structural Features of DJ-1 in Cys106 Oxidation-dependent States

To whom correspondence should be addressed: Dr. Gergely Tóth, MTA-TTK-NAP B - Drug Discovery Research Group - Neurodegenerative Diseases, Institute of Organic Chemistry, Research Center for Natural Sciences, Hungarian Academy of Sciences, Budapest, Hungary; phone: +36 1382 6618, email: toth.gergely@ttk.mta.hu

Keywords: DJ-1; Parkinson disease; oxidative stress; molecular dynamics; CSD; OCS 


\section{Table of Contents}

- Table S1. RMSD values of potential energies.

- Table S2. PDBs used for parametrization.

- Table S3. Hydrodynamic diameter of the X-ray structures of DJ-1.

- Table S4. Occurrences (\% of total simulation time) of individual residues at the monomer-monomer interface of DJ-1.

- Table S5. Per residue contributions to DJ-1 monomer-monomer interface interaction energies calculated by MM-GBSA.

- Table S6 Per residue contributions to DJ-1 monomer-monomer interface interaction energies calculated by MM-GBSA.

- Figure S1. Time evolution of $\mathrm{C} \alpha$ atom root-mean-squared-deviation (RMSD) with a 1ns moving average representation.

- Figure S2. Time evolution of radius of gyration ( $\mathrm{Rg}$ ) with a 1ns moving average representation.

- Figure S3. Time evolution of solvent accessible surface area (SASA) with a 1ns moving average representation.

- Figure S4. Time evolution of MM/GBSA score with a 1ns moving average representation.

- Figure S5. Distances between the backbone O atom of Ser155 and the backbone N atom of Cys106.

- Figure S6. Time evolution of side chain $\chi 1$ angle for chain A (A) and chain B (B) in the reduced, oxidized and over-oxidized DJ-1 simulations.

- Figure S7. Distribution of distances between the side chain S atoms of Cys53 in chain A and chain B.

- Figure S8. Distribution of the hydrodynamic diameter $\left(D_{h}\right)$ in the reduced, oxidized and over-oxidized DJ-1 simulations.

- Figure S9. Reduced, oxidized and over-oxidized DJ-1 monomer-monomer MM-GBSA interaction energy distributions (A) and SASA distributions (B).

- Figure S10. Distances between Pro184 to His126 and Gly159, respectively.

- Figure S11. Helical and unstructured ("loop") secondary structure content of the reduced, oxidized and over-oxidized DJ-1 systems calculated by DSSP.

- $\quad$ Figure S12. Crystal structure of aggregated DJ-1 (PDB ID: 3BWE).

- Figure S13. Hydrodynamic diameter $\left(D_{h}\right)$ of reduced $(A)$, oxidized $(B)$ and over-oxidized (C) DJ-1 determined by DLS.

- Circular dicroism measurement of DJ-1 - Extended Results and Discussion. Figure S14. CD (A) and UV (B) absorption spectra of the reduced (gray) $(30 \mu \mathrm{M})$, oxidized (red) $(31 \mu \mathrm{M})$, and over-oxidized (green) $(33 \mu \mathrm{M})$ form of DJ-1 in the near-UV region. 


\begin{tabular}{l|l|l|l} 
Residue & Conformation & RMSD & \\
\hline CSD $(\mathrm{pot})$ & {$[\mathrm{kcal} / \mathrm{mol}]$} & $\%$ \\
\hline CSD & alpha & 4.25 & 52.2 \\
\hline OCS & extended & 4.73 & 67.8 \\
\hline OCS & alpha & 2.77 & 50.0 \\
\hline ASP & extended & 2.55 & 44.3 \\
\hline ASP & extended & 2.85 & 63.5 \\
\hline HIP & alpha & 2.53 & 57.5 \\
\hline HIP & extended & 1.94 & 47.4 \\
\hline LEU & alpha & 1.30 & 40.4 \\
\hline LEU & extended & 1.08 & 38.2 \\
\hline
\end{tabular}

Table S1. RMSD values of potential energies $\left(\operatorname{RMSD}_{\mathrm{E}(\mathrm{pot})}=\operatorname{SQRT}\left(\left(\Sigma\left(\mathrm{E}_{\mathrm{abinitio,i}} \mathrm{E}_{\mathrm{MM}, \mathrm{i}}\right)^{2}\right) / \mathrm{N}\right)\right)$ and percentage of points exceeding this value are shown for all residues analyzed during the $2 \mathrm{D}$ dihedral scan. 3-sulfinoalanine (CSD) and cysteine sulfonic acid (OCS), aspartate (ASP), protonated histidine (HIP) and leucine (LEU).

\begin{tabular}{l|l}
$\begin{array}{l}\text { CSD containing protein } \\
\text { structures }\end{array}$ & $\begin{array}{l}\text { 1eu1, 1soa, 2r3i, 2zcf, 2zpi, 3f5v, 3sqz, 4n12, 4pst, 4rgc, } \\
\text { 1gz8, 1u9c, 2r3q, 2zpb, 3a8g, 3hht, 4bpy, 4ob0, 1i88, 2cz1, } \\
\text { 2ril, 2zpe, 3a8m, 3hj0, 4ekf, 4oq4, 1oki, 2gcu, 2rk6, 2zpf, } \\
\text { 3a8o, 3mi2, 4ge0, 4p2g, 4ptj, 1qfm, 2qdy, 2wlr, 2zpg, 3ezg, } \\
\text { 3oqp, 4i07, 4pss, 4qyt }\end{array}$ \\
\hline $\begin{array}{l}\text { OCS containing protein } \\
\text { structures }\end{array}$ & $\begin{array}{l}\text { 1j98, 1z70, 2afy, 2v1m, 2wfi, 3r0v, 3ttv, 4d1w, 4lgt } \\
\end{array}$
\end{tabular}

Table S2. PDBs used for parametrization. PDB codes of the protein structures used for parametrization of the modified cysteine residues: 3-sulfinoalanine (CSD) and cysteine sulfonic acid (OCS). 


\begin{tabular}{|l|l|}
\hline PDB code & $\mathbf{D}_{\mathbf{h}}$ of DJ-1 dimer \\
\hline 1J42 & 5.476 \\
\hline 1P5F & 5.606 \\
\hline 1PDV & 5.52 \\
\hline 1PDW_dimer1 & 5.518 \\
\hline 1PDW_dimer2 & 5.48 \\
\hline 1PDW_dimer3 & 5.514 \\
\hline 1PDW_dimer4 & 5.526 \\
\hline 1Q2U & 5.538 \\
\hline 1SOA & 5.618 \\
\hline 1UCF & 5.456 \\
\hline 2OR3 & 5.54 \\
\hline 2R1U & 5.612 \\
\hline 3BWE_dimer1 & 5.494 \\
\hline 3BWE_dimer2 & 5.54 \\
\hline 3BWE_dimer3 & 5.482 \\
\hline 3BWE_dimer4 & 5.46 \\
\hline 4BTE & 5.564 \\
\hline 4P2G & 5.586 \\
\hline 4P34 & 5.602 \\
\hline 4RKW & 5.57 \\
\hline 4ZGG & 5.646 \\
\hline
\end{tabular}

Table S3. Hydrodynamic diameter $\left(D_{h}\right)$ of the analyzed X-ray structures of DJ-1 as calculated by HYDROPRO. 


\begin{tabular}{|r|r|r|r|r|r|r|r|r|}
\hline & & & \multicolumn{6}{|c|}{ Occurrence (\%) } \\
\cline { 5 - 9 } Chain & \multirow{2}{*}{$\begin{array}{r}\text { Residue } \\
\text { name }\end{array}$} & $\begin{array}{c}\text { Residue } \\
\text { number }\end{array}$ & Reduced & Oxidized & Overoxidized & $\begin{array}{l}\text { Delta(Reduced- } \\
\text { Overoxidized) }\end{array}$ & $\begin{array}{l}\text { Delta(Oxidized- } \\
\text { Overoxidized) }\end{array}$ & $\begin{array}{l}\text { Delta } \\
\text { Overage }\end{array}$ \\
\hline B & PRO & 127 & 99.5 & 99.09 & 10.82 & 88.68 & 88.27 & $\mathbf{8 8 . 4 7 5}$ \\
\hline A & LEU & 187 & 100 & 100 & 18.39 & 81.61 & 81.61 & $\mathbf{8 1 . 6 1}$ \\
\hline B & ARG & 156 & 99.97 & 99.96 & 30.63 & 69.34 & 69.33 & $\mathbf{6 9 . 3 3 5}$ \\
\hline B & HIE & 126 & 99.9 & 99.78 & 38.31 & 61.59 & 61.47 & $\mathbf{6 1 . 5 3}$ \\
\hline B & LEU & 128 & 62.07 & 71.39 & 7.4 & 54.67 & 63.99 & $\mathbf{5 9 . 3 3}$ \\
\hline A & LYS & 188 & 77.23 & 72.71 & 21.74 & 55.49 & 50.97 & $\mathbf{5 3 . 2 3}$ \\
\hline A & ALA & 183 & 36.52 & 54.55 & 4.27 & 32.25 & 50.28 & $\mathbf{4 1 . 2 6 5}$ \\
\hline B & LYS & 188 & 76.49 & 85.445 & 64.52 & 11.97 & 20.925 & $\mathbf{1 6 . 4 4 7 5}$ \\
\hline A & GLU & 163 & 20.25 & 28.3 & 11.58 & 8.67 & 16.72 & $\mathbf{1 2 . 6 9 5}$ \\
\hline
\end{tabular}

Table S4. Occurrences (\% of total simulation time) of individual residues at the monomermonomer interface of DJ-1. Residues with at least $10 \%$ Delta Average occurrences are shown. Residues at the C-terminal in chain A and in the His126 region in chain B are colored green and yellow, respectively.

\begin{tabular}{|c|c|c|c|c|c|}
\hline \multirow[b]{2}{*}{ Chain } & \multirow[b]{2}{*}{$\begin{array}{c}\text { Residue } \\
\text { name }\end{array}$} & \multirow[b]{2}{*}{$\begin{array}{l}\text { Residue } \\
\text { number }\end{array}$} & \multicolumn{3}{|c|}{ Per residue monomer-monomer interaction energy } \\
\hline & & & \begin{tabular}{|c|} 
Delta (Reduced- \\
Overoxidized)
\end{tabular} & $\begin{array}{c}\text { Delta (Oxidized- } \\
\text { Overoxidized) }\end{array}$ & \begin{tabular}{|c} 
Delta (Reduced- \\
Oxidized)
\end{tabular} \\
\hline A & GLU & 15 & $\begin{array}{r}-2.681 \\
\end{array}$ & 4.1144 & 0.7167 \\
\hline $\mathrm{A}$ & GLU & 16 & -0.3944 & -1.2126 & -0.8035 \\
\hline A & MET & 17 & 0.1402 & 0.3598 & 0.25 \\
\hline A & GLH & 18 & -0.291 & -0.2554 & -0.2732 \\
\hline A & VAL & 20 & 0.0994 & 0.254 & 0.1767 \\
\hline $\mathrm{A}$ & ILE & 21 & 0.0012 & 0.056 & 0.0286 \\
\hline $\mathrm{A}$ & VAL & 23 & 0.0248 & 0.111 & 0.0679 \\
\hline $\mathrm{A}$ & $\mathrm{ASH}$ & 24 & 0.028 & 0.2416 & 0.1348 \\
\hline $\mathrm{A}$ & VAL & 25 & 0.0058 & 0.0932 & 0.0495 \\
\hline $\mathrm{A}$ & ARG & 27 & 0.2574 & 0.0128 & 0.1351 \\
\hline $\mathrm{A}$ & ARG & 28 & 0.9384 & -1.6784 & -0.37 \\
\hline A & VAL & 35 & 0.0302 & 0.0402 & 0.0352 \\
\hline A & PRO & 43 & -0.0776 & -0.0522 & -0.0649 \\
\hline A & SER & 47 & -0.0854 & 0.1946 & 0.0546 \\
\hline A & ARG & 48 & 0.6138 & -0.0796 & 0.2671 \\
\hline $\mathrm{A}$ & ASP & 49 & -0.7588 & -1.037 & -0.8979 \\
\hline$A$ & VAL & 50 & -0.0576 & -0.0132 & -0.0354 \\
\hline $\mathrm{A}$ & VAL & 51 & -0.0298 & -0.0072 & -0.0185 \\
\hline$A$ & ILE & 52 & -0.0232 & 0.0498 & 0.0133 \\
\hline $\mathrm{A}$ & CYS & 53 & 0.0186 & -0.0102 & 0.0042 \\
\hline $\mathrm{A}$ & ASP & 55 & -0.3212 & 0.053 & -0.1341 \\
\hline A & CYM & 106 & -0.1684 & -1.5702 & -0.8693 \\
\hline A & HIE & 126 & 0.1808 & 0.3992 & 0.29 \\
\hline $\mathrm{A}$ & PRO & 127 & 0.3296 & 0.2698 & 0.2997 \\
\hline $\mathrm{A}$ & LEU & 128 & 0.5326 & 0.5836 & 0.5581 \\
\hline $\mathrm{A}$ & ARG & 145 & 4.9712 & 2.2368 & 3.604 \\
\hline $\mathrm{A}$ & ARG & 156 & 3.4496 & 2.0774 & 2.7635 \\
\hline $\mathrm{A}$ & GLY & 157 & 0.1214 & -0.244 & -0.0613 \\
\hline $\mathrm{A}$ & PRO & 158 & 0.0868 & 0.0188 & 0.0528 \\
\hline $\mathrm{A}$ & GLY & 159 & -0.164 & -0.7588 & -0.4614 \\
\hline A & THR & 160 & 0.0106 & -0.2914 & -0.1404 \\
\hline A & SER & 161 & 0.384 & 0.4226 & 0.4033 \\
\hline A & PHE & 162 & 0.9144 & 0.9884 & 0.9514 \\
\hline A & GLU & 163 & -3.519 & -4.371 & -3.945 \\
\hline A & ALA & 183 & -0.1528 & -0.347 & -0.2499 \\
\hline A & PRO & 184 & 2.1562 & 2.0792 & 2.1177 \\
\hline$A$ & LEU & 185 & -4.7992 & -4.8136 & -4.8064 \\
\hline A & VAL & 186 & -5.6214 & -5.1156 & -5.3685 \\
\hline A & LEU & 187 & -2.0012 & -1.3948 & -1.698 \\
\hline $\mathrm{A}$ & LYS & 188 & -7.2462 & -6.7032 & -6.9747 \\
\hline
\end{tabular}

\begin{tabular}{|c|c|c|c|c|c|}
\hline \multirow[b]{2}{*}{ Chain } & \multirow[b]{2}{*}{$\begin{array}{c}\text { Residue } \\
\text { name }\end{array}$} & \multirow{2}{*}{$\begin{array}{l}\text { Residue } \\
\text { number }\end{array}$} & \multicolumn{3}{|c|}{ Per residue monomer-monomer interaction energy } \\
\hline & & & \begin{tabular}{|c|} 
Delta (Reduced- \\
Overoxidized)
\end{tabular} & \begin{tabular}{|c|} 
Delta (Oxidized- \\
Overoxidized)
\end{tabular} & $\begin{array}{c}\text { Delta (Reduced- } \\
\text { Oxidized) }\end{array}$ \\
\hline$B$ & GLU & 15 & 2.014 & 1.8592 & 1.9366 \\
\hline$B$ & GLU & 16 & 1.4734 & 2.3664 & 1.9199 \\
\hline$B$ & MET & 17 & 0.1818 & 0.1944 & 0.1881 \\
\hline$B$ & GLH & 18 & -0.6416 & -0.2998 & -0.4707 \\
\hline$B$ & VAL & 20 & -0.1272 & -0.3374 & -0.2323 \\
\hline$B$ & ILE & 21 & 0.0126 & 0.0068 & 0.0097 \\
\hline B & VAL & 23 & -0.2058 & -0.3914 & -0.2986 \\
\hline B & ASH & 24 & -0.3042 & -1.8314 & -1.0678 \\
\hline$B$ & VAL & 25 & -0.2196 & -0.2994 & -0.2595 \\
\hline$B$ & ARG & 27 & -0.9704 & -1.4814 & -1.2259 \\
\hline$B$ & ARG & 28 & -1.2142 & 4.6084 & 1.6971 \\
\hline$B$ & VAL & 35 & -0.0664 & -0.0766 & -0.0715 \\
\hline$B$ & PRO & 43 & 0.0564 & 0.0294 & 0.0429 \\
\hline$B$ & SER & 47 & -0.1652 & -0.2362 & -0.2007 \\
\hline$B$ & ARG & 48 & \begin{tabular}{|l|l|}
-482 \\
\end{tabular} & -1.5654 & -1.5237 \\
\hline$B$ & ASP & 49 & 0.6108 & 1.2192 & 0.915 \\
\hline$B$ & VAL & 50 & 0.0022 & -0.0806 & -0.0392 \\
\hline$B$ & VAL & 51 & -0.0104 & 0.0922 & 0.0409 \\
\hline B & ILE & 52 & 0.004 & 0.0122 & 0.0081 \\
\hline$B$ & CYS & 53 & -0.0612 & -0.0304 & -0.0458 \\
\hline B & ASP & 55 & 0.1382 & 0.5704 & 0.3543 \\
\hline B & CYM & 106 & 5.514 & 3.135 & 4.3245 \\
\hline B & HIE & 126 & -4.4128 & -4.3676 & -4.3902 \\
\hline B & PRO & 127 & -0.764 & -0.8588 & -0.8114 \\
\hline$B$ & LEU & 128 & -0.3958 & -0.433 & -0.4144 \\
\hline$B$ & ARG & 145 & -1.9834 & -2.0642 & -2.0238 \\
\hline$B$ & ARG & 156 & -3.156 & -3.5102 & -3.3331 \\
\hline$B$ & GLY & 157 & -0.6796 & -0.6824 & -0.681 \\
\hline$B$ & PRO & 158 & -1.499 & -1.8622 & -1.6806 \\
\hline$B$ & GLY & 159 & -0.9004 & -0.675 & -0.7877 \\
\hline$B$ & THR & 160 & -0.6868 & -0.5916 & -0.6392 \\
\hline$B$ & SER & 161 & -0.383 & -0.4058 & -0.3944 \\
\hline B & PHE & 162 & -0.0382 & -0.0998 & -0.069 \\
\hline$B$ & GLU & 163 & -2.1428 & -0.1096 & -1.1262 \\
\hline$B$ & ALA & 183 & 0.353 & 0.2242 & 0.2886 \\
\hline B & PRO & 184 & 0.0732 & -0.512 & -0.2194 \\
\hline$B$ & LEU & 185 & 0.1816 & -0.1358 & 0.0229 \\
\hline$B$ & VAL & 186 & 0.0926 & -0.8564 & -0.3819 \\
\hline$B$ & LEU & 187 & 0.593 & 0.047 & 0.32 \\
\hline$B$ & LYS & 188 & -1.888 & -5.8696 & -3.8788 \\
\hline
\end{tabular}

Table S5. Per residue contributions to DJ-1 monomer-monomer interface interaction energies calculated by MM-GBSA. Residues with largest contributions at the C-terminal in chain A and in the His 126 region in chain B are colored green and yellow, respectively. 


\begin{tabular}{|c|c|c|c|c|c|c|c|c|}
\hline \multirow{2}{*}{ Chain } & \multirow{2}{*}{$\begin{array}{c}\text { Residue } \\
\text { name }\end{array}$} & \multirow{2}{*}{$\begin{array}{l}\text { Residue } \\
\text { number }\end{array}$} & \multicolumn{6}{|c|}{ Per residue solvent accessible surface area $\left(\mathrm{nm}^{2}\right)$} \\
\hline & & & Reduced & Oxidized & Overoxidized & \begin{tabular}{|c|} 
Delta (Reduced- \\
Overoxidized)
\end{tabular} & \begin{tabular}{|c|} 
Delta (Oxidized- \\
Overoxidized)
\end{tabular} & $\begin{array}{c}\text { Delta } \\
\text { (Average) }\end{array}$ \\
\hline $\mathrm{B}$ & TYR & 141 & 0.354 & 0.388 & 0.765 & $\begin{array}{r}-0.411 \\
\end{array}$ & $\begin{array}{r}-0.377 \\
\end{array}$ & -0.394 \\
\hline B & HIE & 126 & 0.163 & 0.128 & 0.539 & -0.376 & -0.411 & -0.3935 \\
\hline A & VAL & 186 & 0.299 & 0.273 & 0.624 & -0.325 & -0.351 & -0.338 \\
\hline $\mathrm{B}$ & ARG & 156 & 0.244 & 0.225 & 0.506 & -0.262 & -0.281 & -0.2715 \\
\hline $\mathrm{A}$ & LYS & 188 & 2.321 & 2.266 & 2.525 & -0.204 & -0.259 & -0.2315 \\
\hline B & PRO & 127 & 0.629 & 0.596 & 0.832 & -0.203 & -0.236 & -0.2195 \\
\hline $\mathrm{B}$ & LEU & 151 & 0.5 & 0.347 & 0.577 & -0.077 & -0.23 & -0.1535 \\
\hline $\mathrm{A}$ & ARG & 5 & 0.735 & 0.765 & 0.901 & -0.166 & -0.136 & -0.151 \\
\hline $\mathrm{B}$ & GLU & 143 & 1.199 & 1.186 & 1.335 & -0.136 & -0.149 & -0.1425 \\
\hline $\mathrm{A}$ & ALA & 129 & 0.059 & 0.065 & 0.203 & -0.144 & -0.138 & -0.141 \\
\hline B & GLY & 137 & 0.657 & 0.63 & 0.77 & -0.113 & -0.14 & -0.1265 \\
\hline
\end{tabular}

Table S6. Per residue contributions to DJ-1 monomer-monomer interface interaction energies calculated by MM-GBSA. Residues with largest contributions at the C-terminal in chain A and in the His 126 region in chain B are colored green and yellow, respectively. Residues with at least $0.1 \%$ Delta Average are shown.

\section{Ca RMSD Evolution with 1ns Moving Averages}

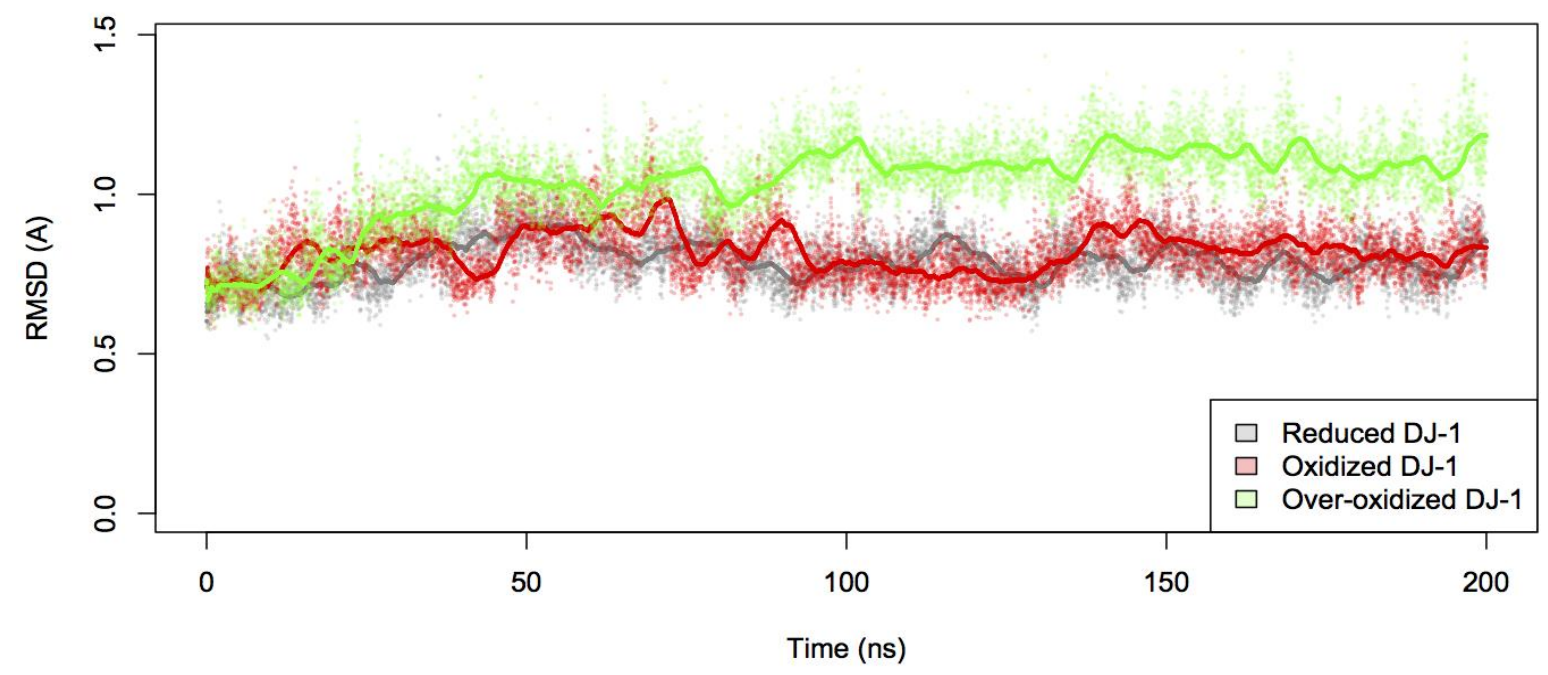


Figure S1. Time evolution of $\mathrm{C} \alpha$ atom RMSD with a 1ns moving average representation.

Radius of Gyration (Rg) Evolution with 1ns Moving Averages

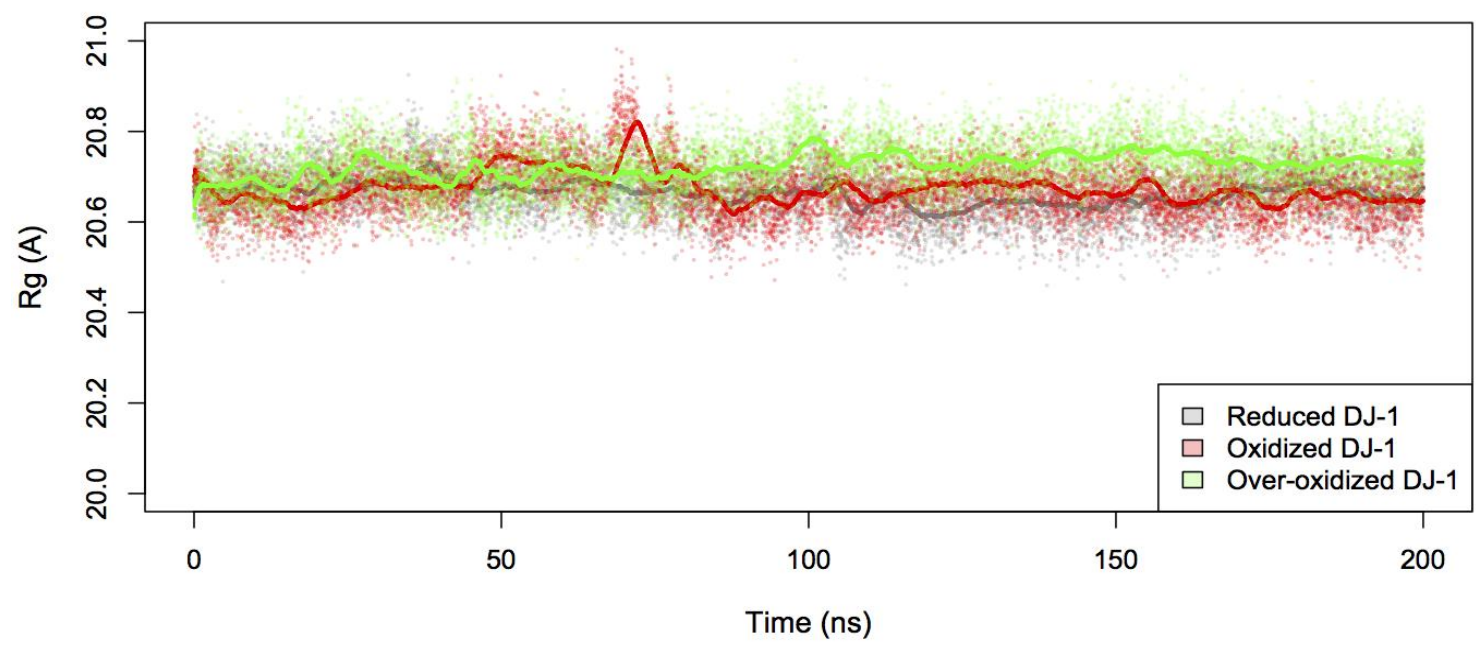

Figure S2. Time evolution of radius of gyration $(\mathrm{Rg})$ with a $1 \mathrm{~ns}$ moving average representation.

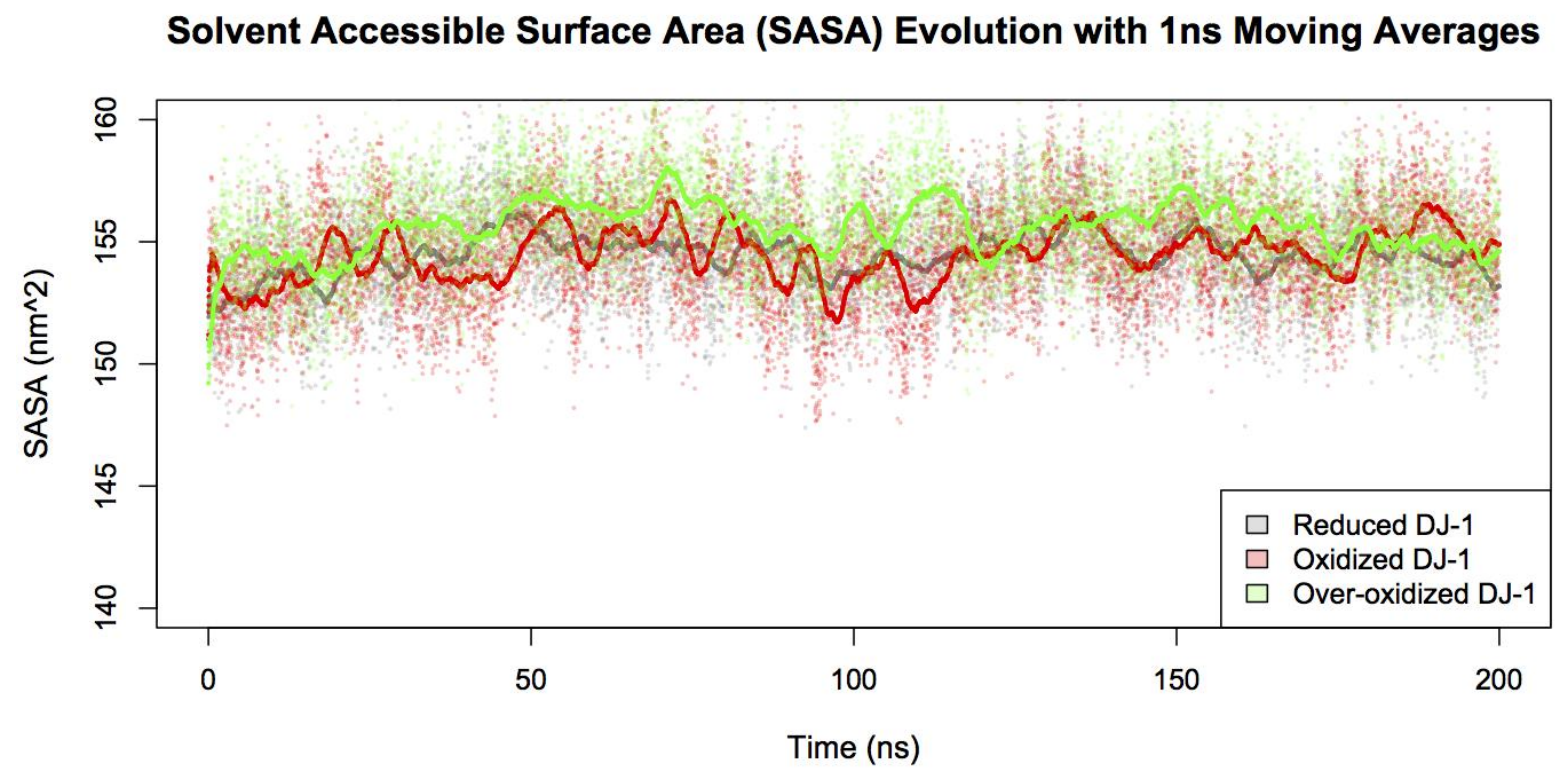

Figure S3. Time evolution of solvent accessible surface area (SASA) with a 1ns moving average representation. 


\section{MM/GBSA Evolution with 1ns Moving Averages}

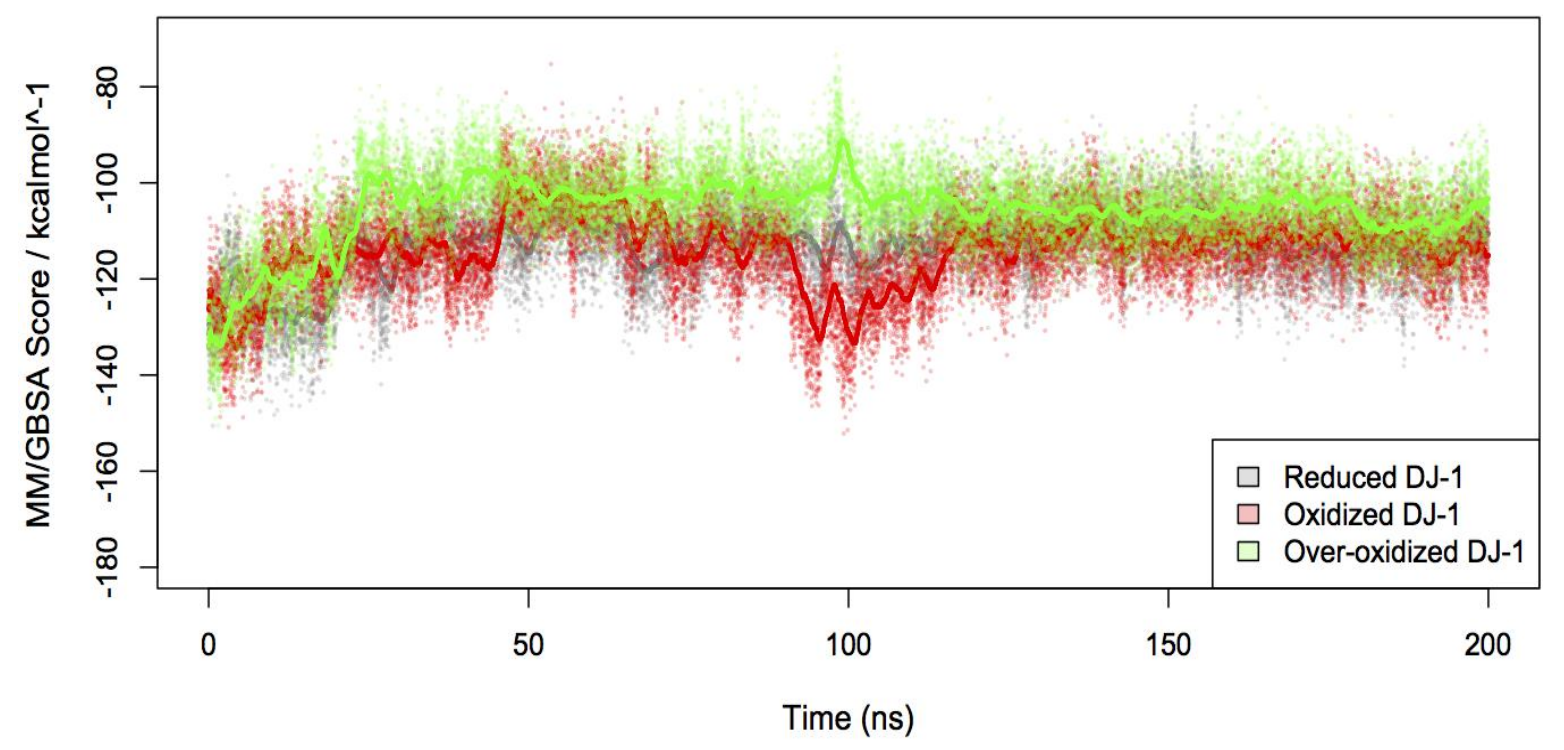

Figure S4. Time evolution of MM/GBSA score with a 1ns moving average representation. 


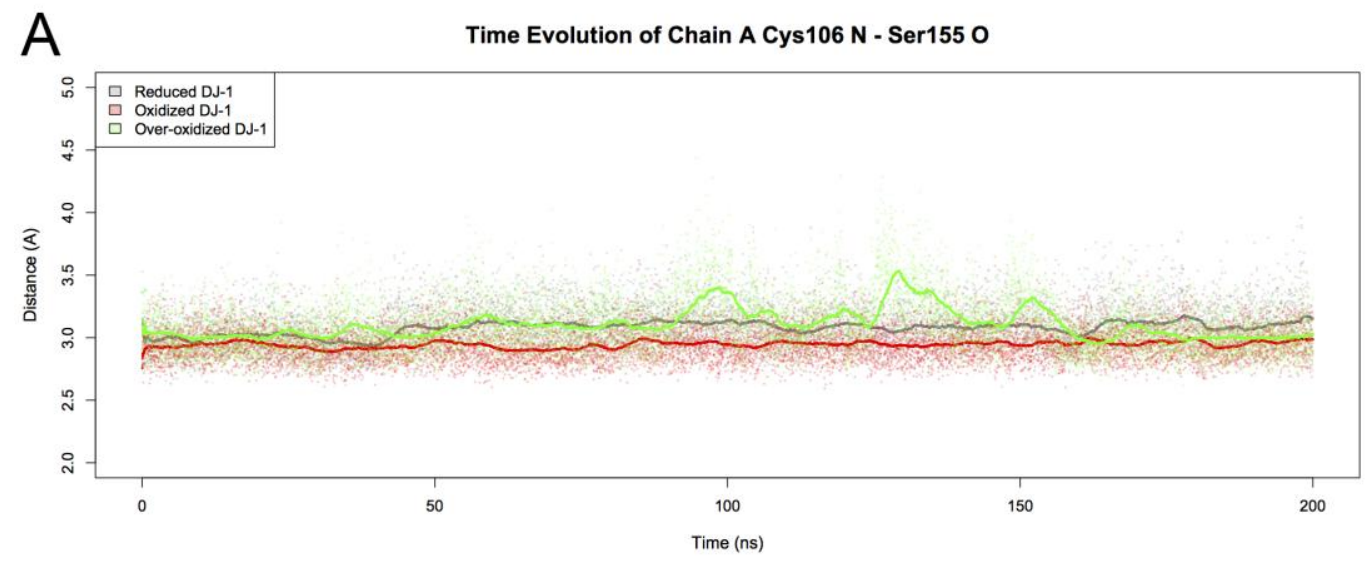

B
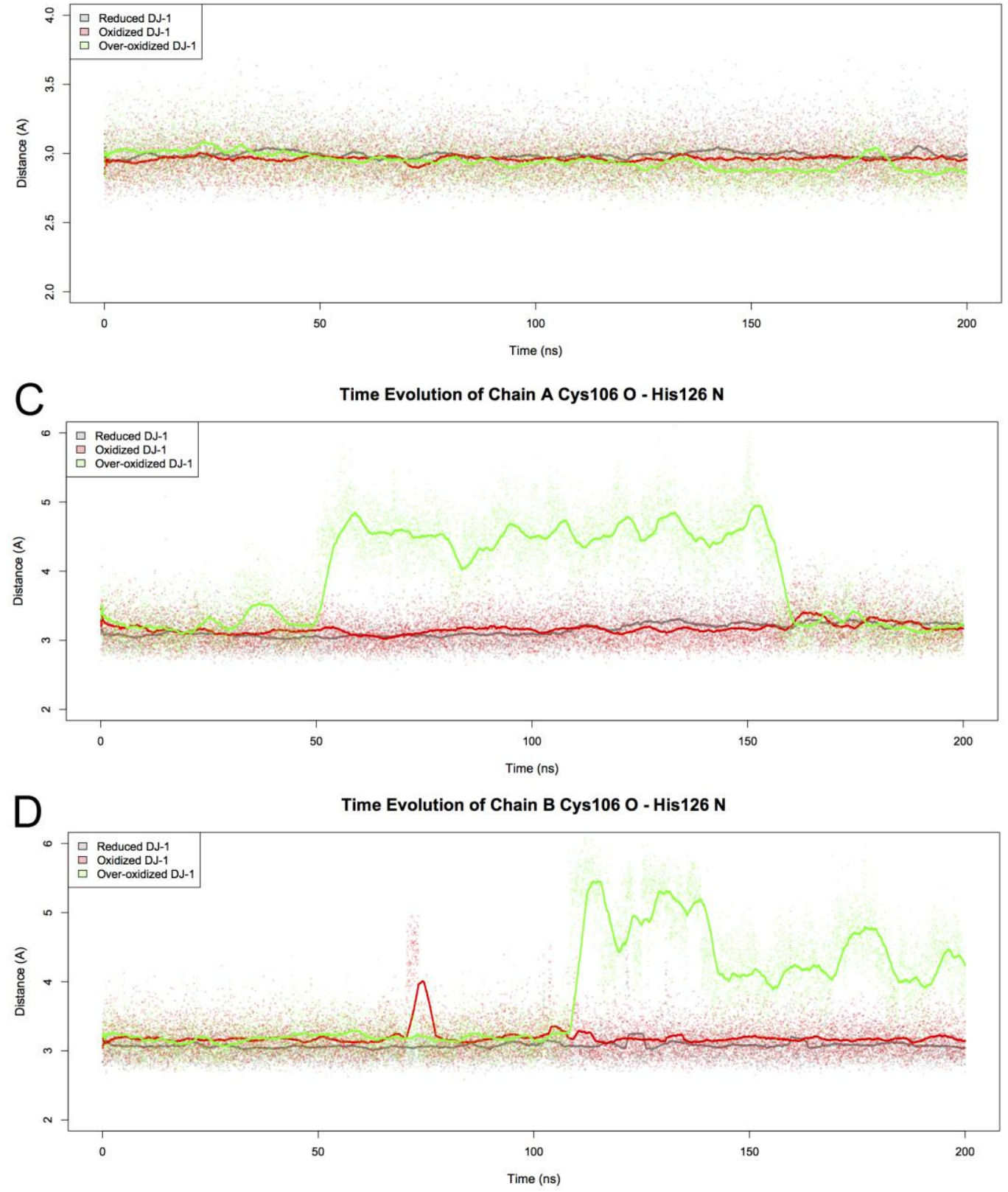
Figure S5. Distances between the backbone O atom of Ser155 and the backbone $N$ atom of Cys106 (A, B). Distances between the backbone $\mathrm{N}$ atom of His 126 and the backbone $\mathrm{O}$ atom of Cys106 (C, D). Distances for chain A (A, C) and chain B (B, D) are shown.

\section{A Chain A Cys106 Chi Angle Evolution}

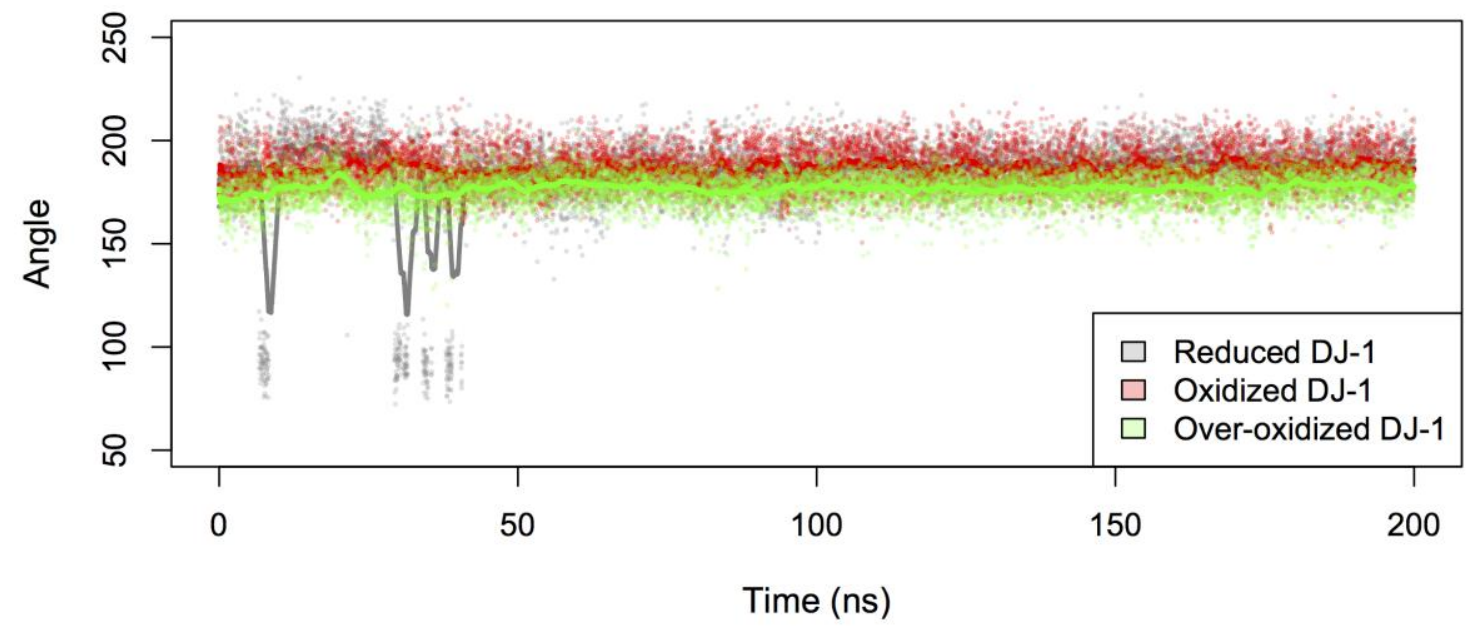

B Chain B Cys106 Chi Angle Evolution

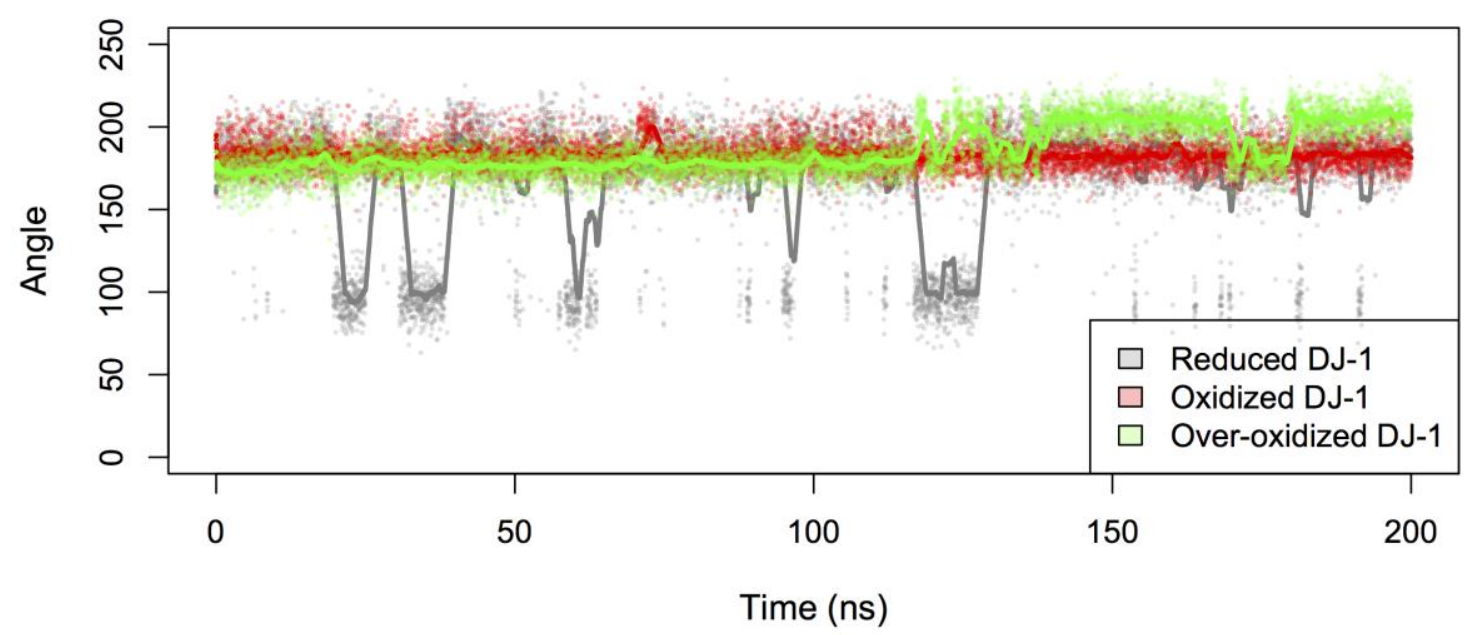

Figure S6. Time evolution of side chain $\chi 1$ angle for chain A (A) and chain B (B) in the reduced, oxidized and over-oxidized DJ-1 simulations. 


\section{Chain A Cys53 SG to Chain B Cys53 SG Distance Distribution}

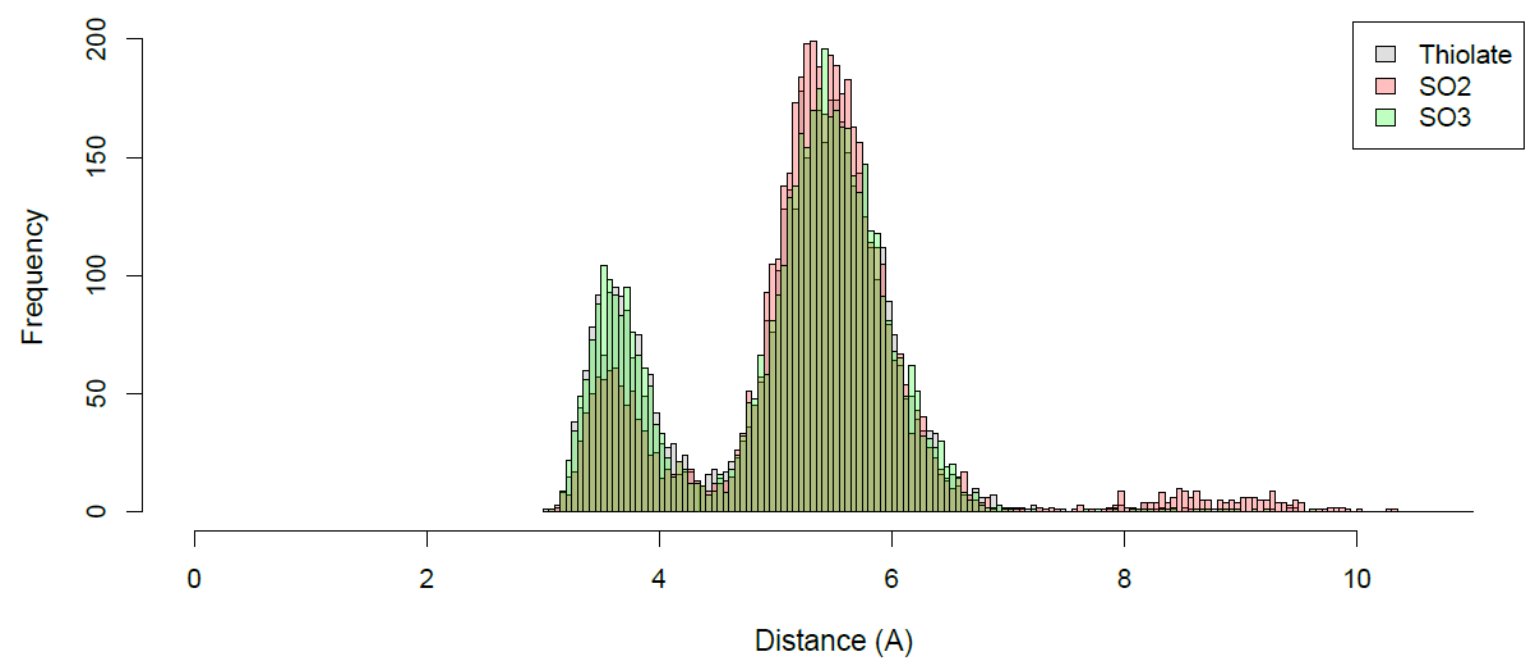

Figure S7. Distribution of distances between the side chain S atoms of Cys53 in chain A and chain B.

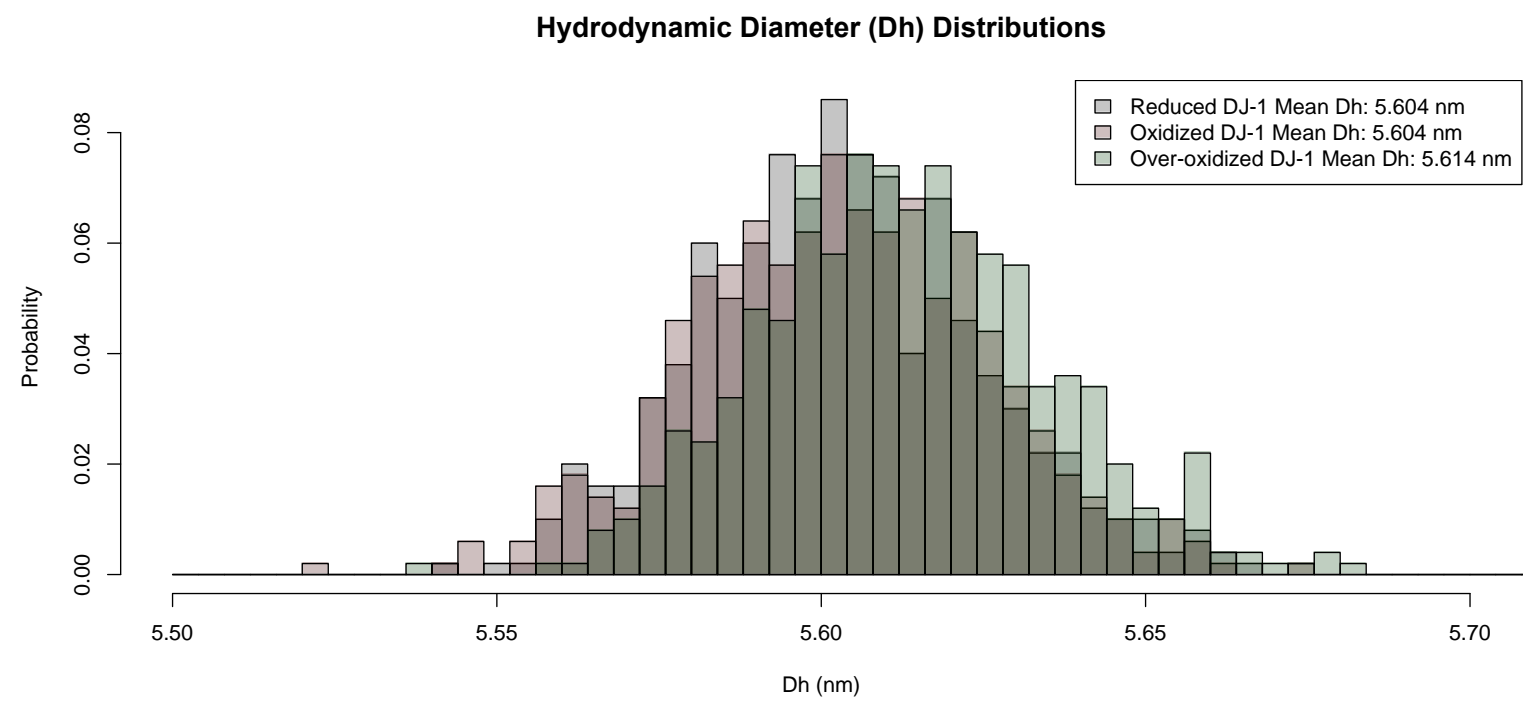

Figure S8. Distribution of the hydrodynamic diameter $\left(D_{h}\right)$ in the reduced, oxidized and overoxidized DJ-1 simulations. 

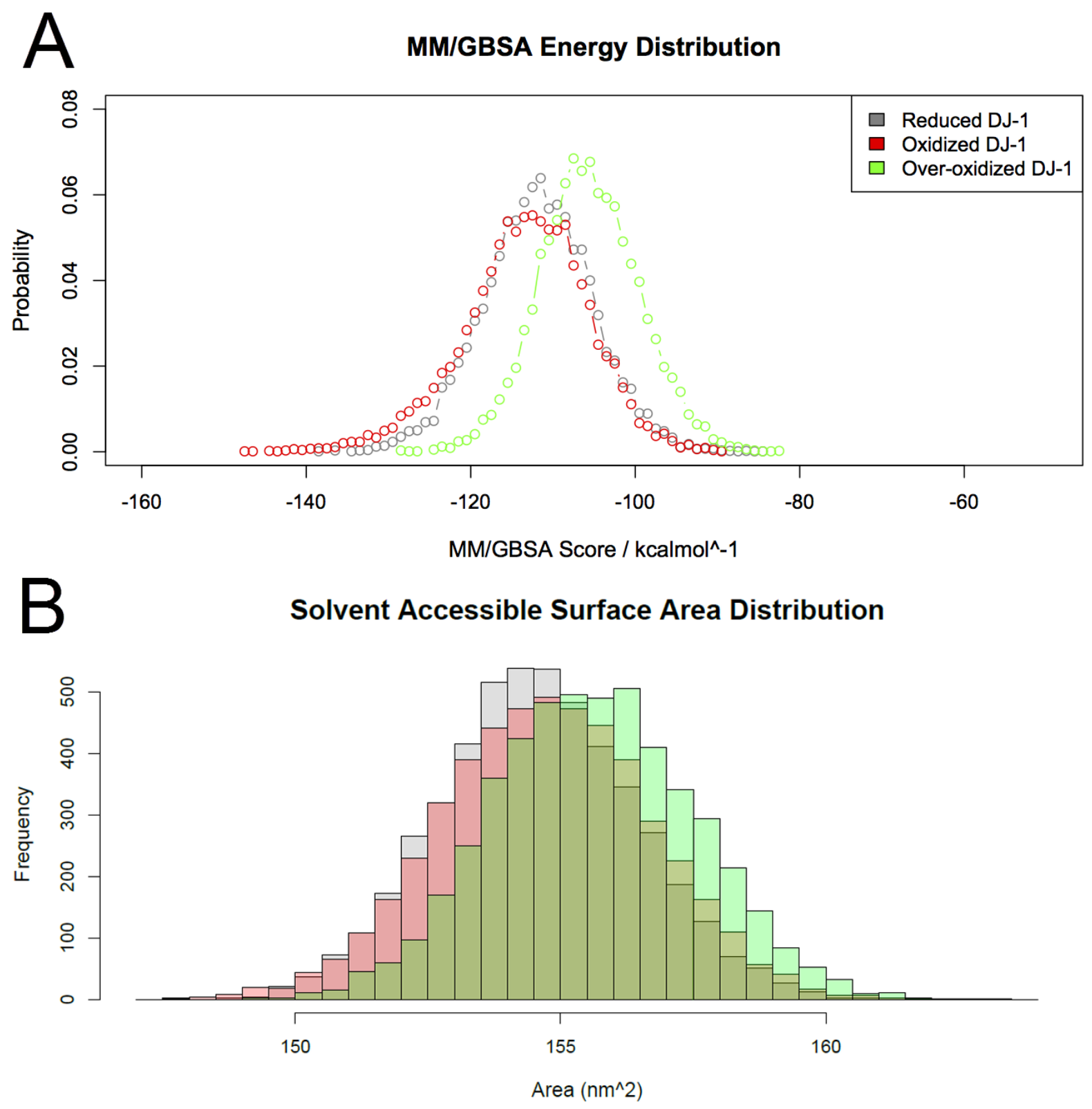

Figure S9. Reduced, oxidized and over-oxidized DJ-1 monomer-monomer MM-GBSA interaction energy (A) and SASA distributions (B). 

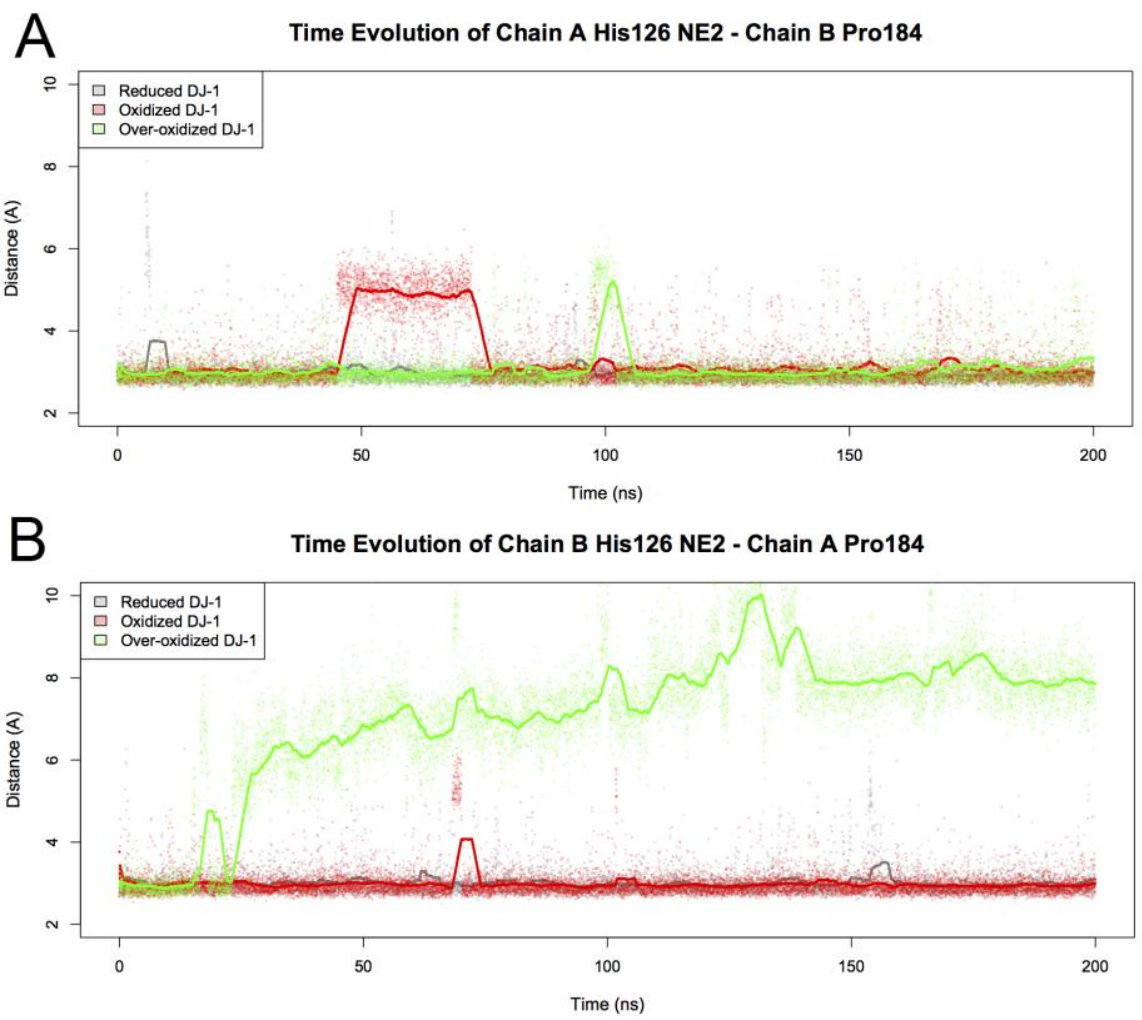

C Time Evolution of Chain A Gly159 N - Chain B Pro184 O

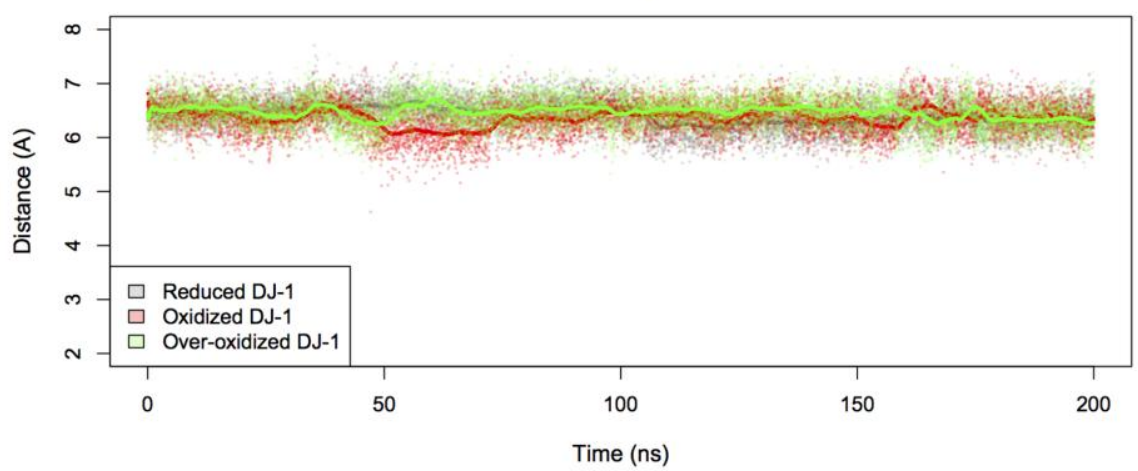

D Time Evolution of Chain B Gly159 N - Chain A Pro184 O

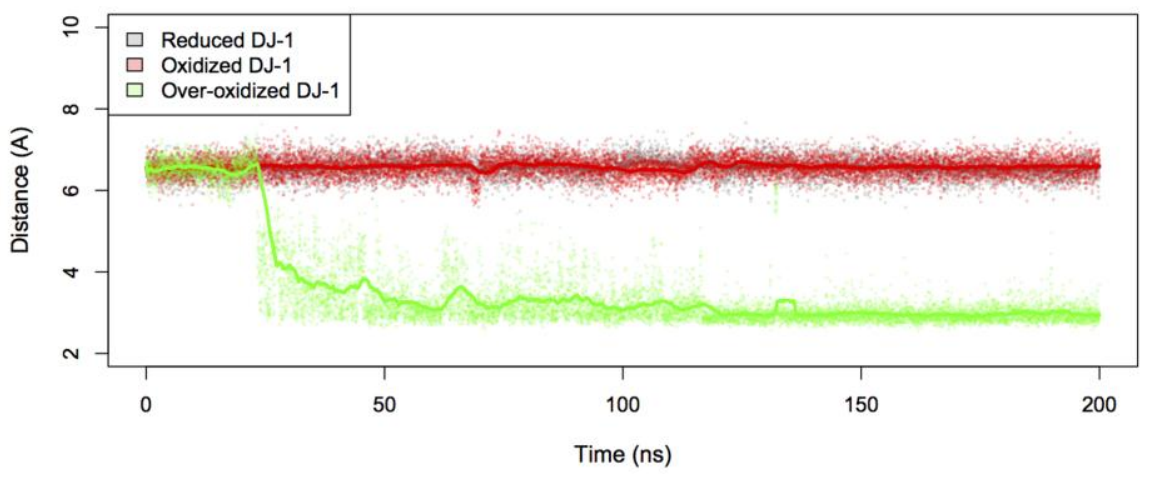


Figure S10. Distances between the side chain $\mathrm{N}(\mathrm{H})$ atom of His126 in chain A and the backbone $\mathrm{O}$ atom of Pro184 in chain B (A). Distances between the side chain $\mathrm{N}(\mathrm{H})$ atom of His126 in chain B and the backbone $\mathrm{O}$ atom of Pro184 in chain A (B). Distances between the backbone N atom of Gly159 in chain A and the backbone $O$ atom of Pro184 in chain B (C). Distances between the backbone $\mathrm{N}$ atom of Gly159 in chain B and the backbone $\mathrm{O}$ atom of Pro184 in chain A (D).

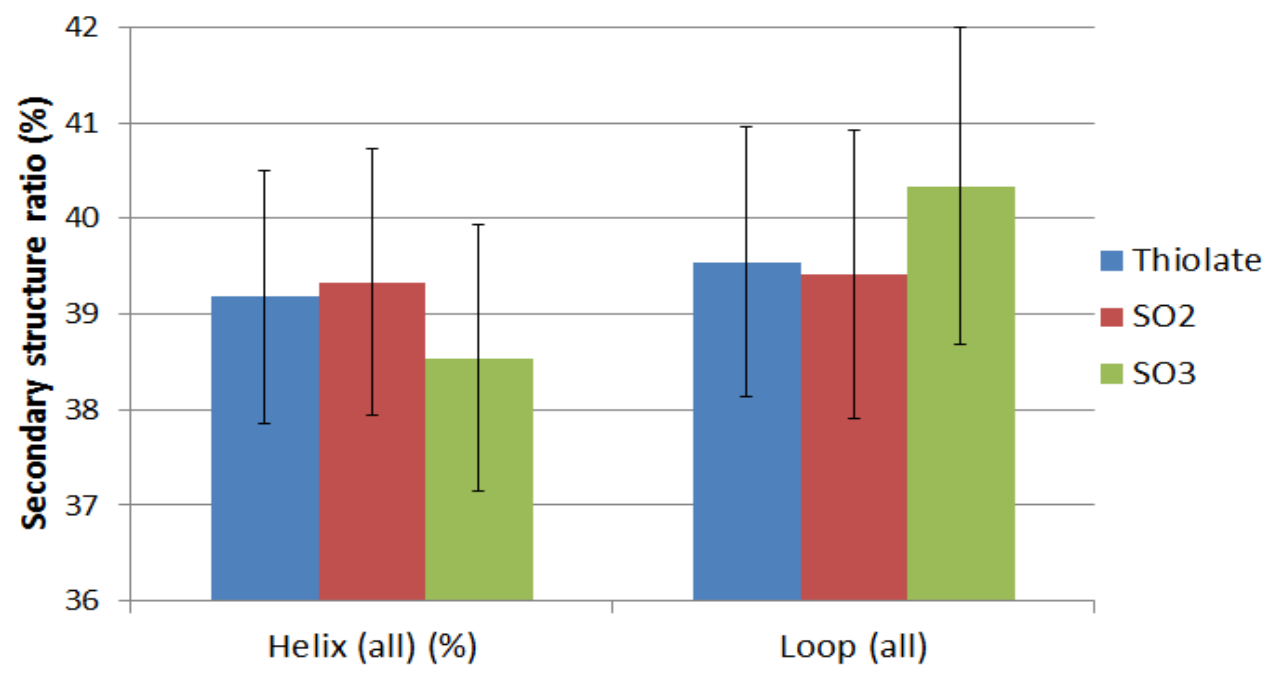

Figure S11. Helical and unstructured ("loop") secondary structure content of the reduced, oxidized and over-oxidized DJ-1 calculated by DSSP. 

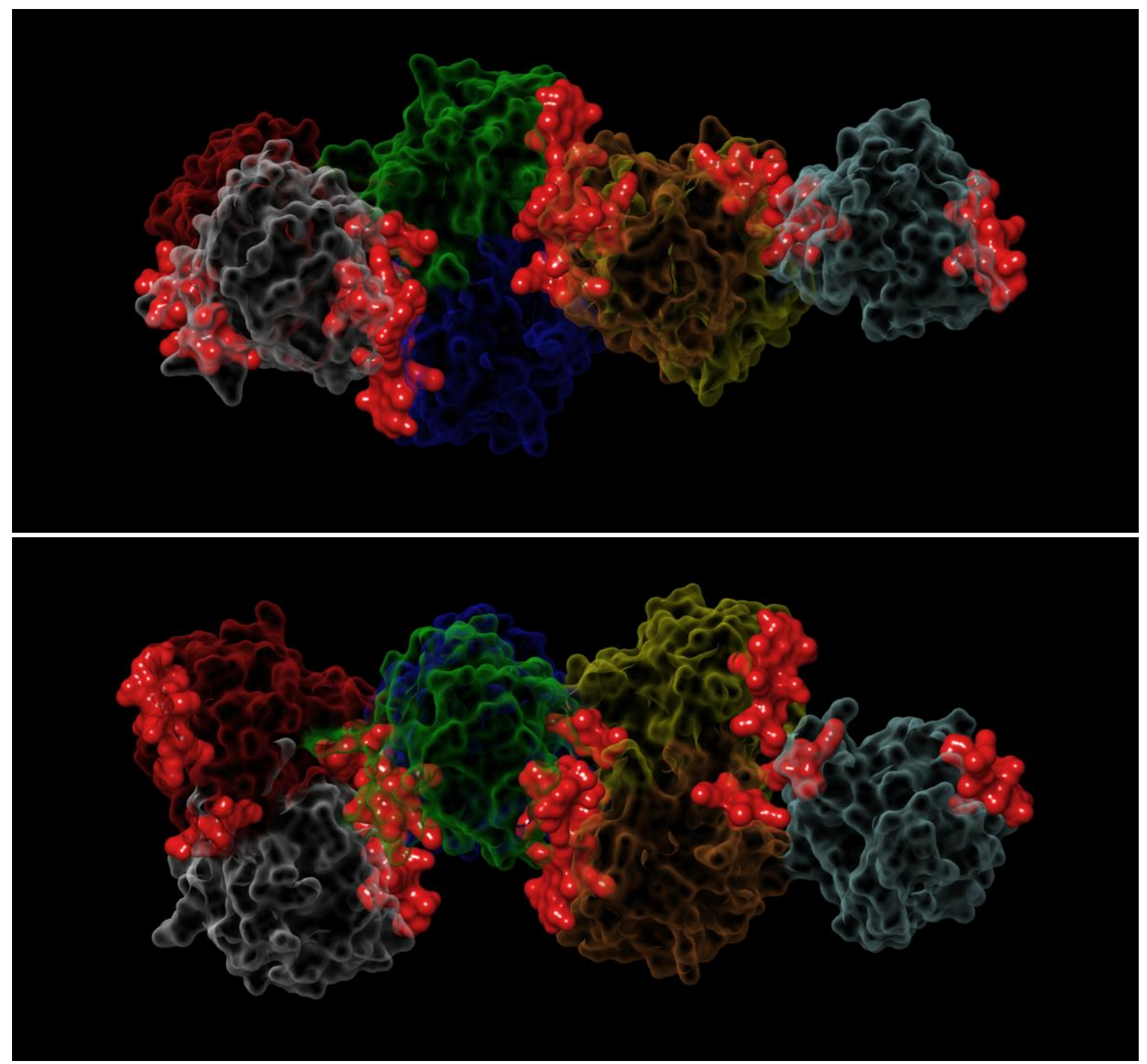

Figure S12. Crystal structure of aggregated DJ-1 (PDB ID: 3BWE). Individual monomers are colored differently. Regions with decreased helical secondary structure in over-oxidized DJ-1 simulations are indicated with red, filled surfaces. 
(A)

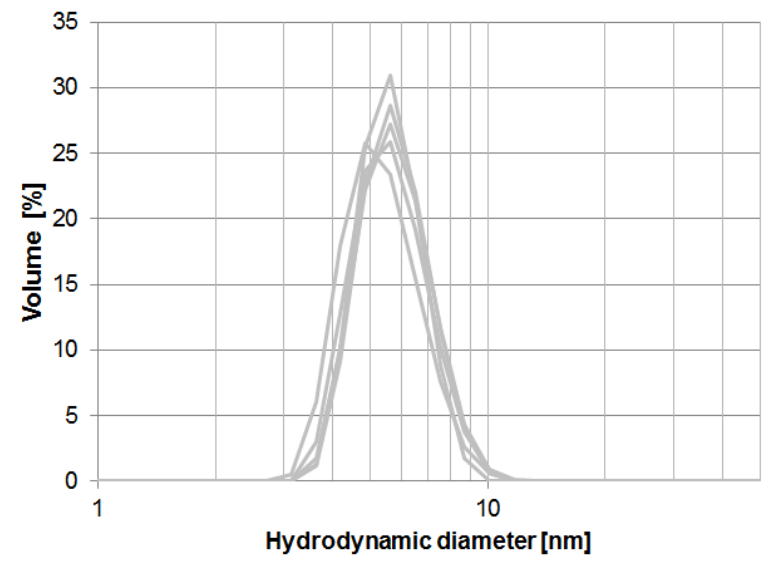

(B)

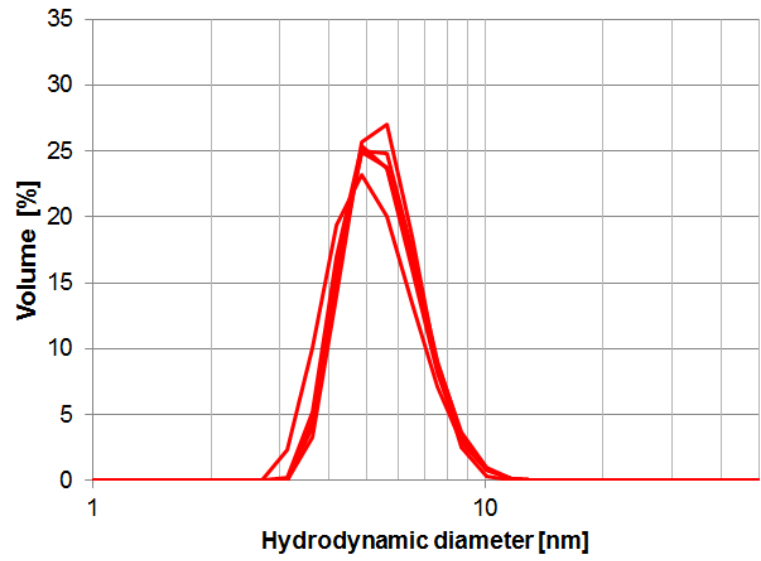

(C)

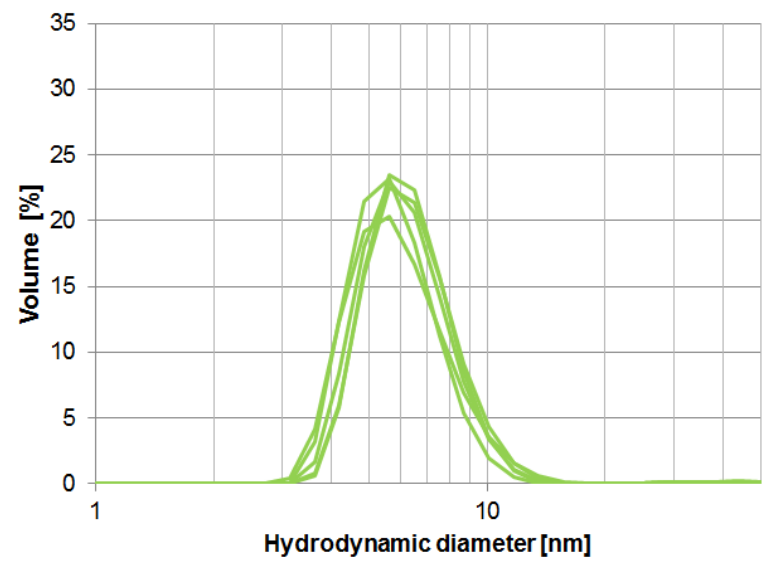

Figure S13. : Hydrodynamic diameter $\left(\mathrm{D}_{\mathrm{h}}\right)$ of reduced $(A)$, oxidized $(B)$ and over-oxidized (C) DJ-1 determined by DLS. 


\section{Spectroscopic Measurements of DJ-1 - Extended Results and Discussion}

Far-UV CD spectra of the reduced and oxidized forms of DJ-1 exhibit two distinctive extrema (Fig. 7B, main article). The minimum at $219 \mathrm{~nm}$ stems from the $\mathrm{n}-\pi^{*}$ transitions of the carbonyl groups while the second extremum around $211 \mathrm{~nm}$ corresponds to the negative branch of the exciton CD couplet of amide $\pi-\pi^{*}$ transitions. The positive exciton CD peak below $200 \mathrm{~nm}$ could not be measured due to the very strong UV absorption of the buffer component dithiothreitol. The double ellipticity minimum in the CD curves at 211 and $219 \mathrm{~nm}$ is a characteristic sign for the significant extent of $\alpha$-helical content. It is to be noted that in helical polypeptides the $\pi-\pi^{*}$ and $n-\pi^{*}$ bands are at 208 and $220 \mathrm{~nm}$, respectively. The red and blue shift of these peaks in the CD spectrum of DJ-1 might be due to the spectral overlap with the $218 \mathrm{~nm}$ negative band of the significant $\beta$-sheet content [2,3] which is supported by the secondary structure analysis (see inset in Fig. 7B, main article). The high qualitative and quantitative similarities between the CD curves of the reduced and oxidized samples indicates that chemical modification of the Cys106 residue does not affect the secondary structure of DJ-1. This conclusion is in a full concordance with the percentages of secondary structure elements calculated from the $\mathrm{CD}$ data which was found to be identical for each sample (Fig. 7B, main article). Deconvolution of the spectra employing the K2d algorithm predicted a $37 \%$-helix content that well agrees with previous CD spectroscopic results [3].
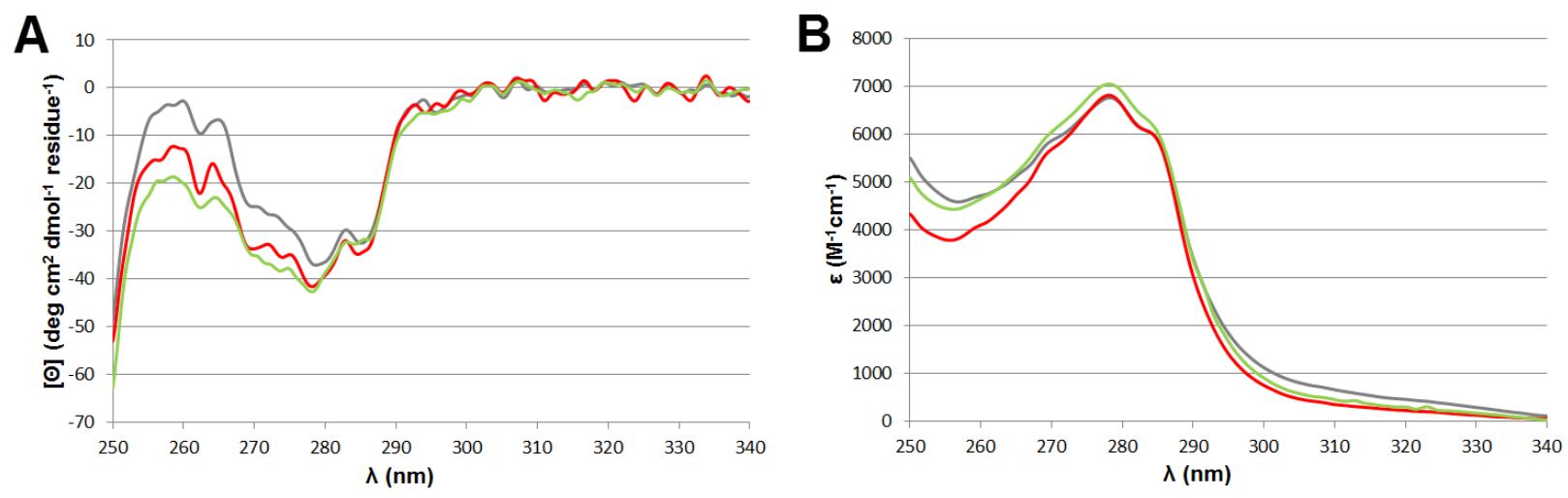

Figure S14. CD (A) and UV (B) absorption spectra of the reduced (gray) (30 $\mu \mathrm{M}$ ), oxidized (red) (31 $\mu \mathrm{M})$, and over-oxidized (green) $(33 \mu \mathrm{M})$ form of DJ-1 in the near-UV region. 
The near-UV CD spectrum of proteins is dominated by $\pi-\pi^{*}$ transitions of aromatic sidechains and can be used as the fingerprint region of the tertiary structure. The CD signals measured here sensitively probe local structural changes affecting the molecular environment and conformation of the aromatic rings. DJ-1 contains three Phe and three Tyr but not Trp residues. In general, CD activity of Tyr is much larger than that of Phe. Therefore, the broad negative band displayed above $265 \mathrm{~nm}$ can be assigned to the ${ }^{1} L_{\mathrm{b}}$ contribution of the Tyr residues (Fig. S14). It exhibits a characteristic vibrational fine structure, the shape of which as well as the position of the vibronic sub-bands are in close correlation with that of the UV absorption band.

In relation to the phenolic ring of Tyr, the ${ }^{1} L_{\mathrm{b}}$ transition of the phenyl chromophore of Phe is at shorter wavelengths $\left(\varepsilon_{\max } \approx 200 \mathrm{M}^{-1} \mathrm{~cm}^{-1}\right.$ at $\left.260 \mathrm{~nm}\right)$. Accordingly, the weaker negative signal at $262.4 \mathrm{~nm}$ is associated to the asymmetrically perturbed ${ }^{1} L_{\mathrm{b}}$ transition of the Phe side-chains [4]. Upon oxidation of the Cys106 residue, amplitudes of the near-UV CD signals significantly increase below $280 \mathrm{~nm}$ (Fig. S14). These intensity changes, however, are inhomogeneous in nature showing a gradual enhancement toward shorter wavelengths. The intensification is most pronounced at the $262 \mathrm{~nm}$ peak, the molar ellipticity value of which rises from -9.6 to -22 and 25 , respectively. In contrast, a much smaller intensity increase can be seen for the Tyr allied ellipticity minimum around $278 \mathrm{~nm}$ (Fig. S14). This discrepancy suggests the more prominent role of the Phe residues in relation to tyrosines in the $\mathrm{CD}$ spectral changes. Presumably via allosteric mechanisms, oxidation of Cys106 results in a sterically more constrained environment around the Phe side-chains. The decrease of the conformational freedom, i.e. the rotation of the phenyl ring(s) reduces the number of rotameric species having opposite rotatory strength and thus gives rise to enhanced CD activity [4].

\section{References}

1. Andrade MA, Chacón P, Merelo JJ, Morán F. Evaluation of secondary structure of proteins from UV circular dichroism spectra using an unsupervised learning neural network. Protein Eng. 1993 (6) 383390.

2. Tao X, Tong L. Crystal structure of human DJ-1, a protein associated with early onset Parkinson's disease. J. Biol. Chem. 2003 (278) 31372-31379. 
3. Görner K, Holtorf E, Odoy S, Nuscher B, Yamamoto A, Regula JT, Beyer K, Haass C, Kahle PJ. Differential effects of Parkinson's disease-associated mutations on stability and folding of DJ-1. J. Biol. Chem. 2004 (279) 6943-6951.

4. Strickland EH. Aromatic contributions to circular dichroism spectra of proteins. CRC Crit. Rev. Biochem. 1974 (2) 113-175. 